\title{
The heart rate discrimination task: a psychophysical method to estimate the accuracy and precision of interoceptive beliefs
}

\author{
Nicolas Legrand ${ }^{1}$, Niia Nikolova ${ }^{1}$, Camile Correa $^{1}$, Malthe Brændholt ${ }^{1}$, Anna Stuckert $^{1}$, \\ Nanna Kildahl ${ }^{1}$, Melina Vejlø $\varnothing^{1}$, Francesca Fardo ${ }^{1,2}$, Micah Allen ${ }^{1,3,4}$ \\ ${ }_{1}$ Center of Functionally Integrative Neuroscience, Aarhus University Hospital, Denmark \\ ${ }_{2}$ Danish Pain Research Center \\ ${ }_{3}$ Aarhus Institute of Advanced Studies, Aarhus University, Denmark \\ ${ }_{4}$ Cambridge Psychiatry, University of Cambridge, United Kingdom
}

\begin{abstract}
(150 words):
Interoception - the physiological sense of our inner bodies - has risen to the forefront of psychological and psychiatric research. Much of this research utilizes tasks that attempt to measure the ability to accurately detect cardiac signals. Unfortunately, these approaches are confounded by well-known issues limiting their validity and interpretation. At the core of this controversy is the role of subjective beliefs about the heart rate in confounding measures of interoceptive accuracy. Here, we recast these beliefs as an important part of the causal machinery of interoception, and offer a novel psychophysical "heart rate discrimination" method to estimate their accuracy and precision. By applying this task in 218 healthy participants, we demonstrate that cardiac interoceptive beliefs are more biased, less precise, and are associated with poorer metacognitive insight relative to a matched exteroceptive control. Our task, provided as an open-source python package, offers users an intuitive, robust approach to quantifying cardiac beliefs.
\end{abstract}

\section{Highlights (85 chars each):}

- Current interoception tasks conflate cardiac beliefs with accuracy.

- We introduce a Bayesian method for estimating cardiac belief accuracy and precision.

- Individuals underestimate their heart rate by -7 BPM (95\% CI [-8.6 -5.3]) on average.

- Cardiac beliefs are associated with reduced precision and metacognitive insight.

- The task and modelling tools are provided in the Python Cardioception Package.

keywords: heart rate discrimination, heartbeat tracking, interoception, psychophysics, metacognition 


\section{Introduction}

Interoception denotes the ability to sense, perceive, and control internal visceral states (Chen et al., 2021; Sherrington, 1952). This ability is thought to depend on unique neurobiological pathways, which underpin the affective and somatic axes of selfhood (Craig, 2002; Critchley \& Garfinkel, 2017; Seth \& Tsakiris, 2018; Strigo \& Craig, 2016). Measuring the individual interoceptive capacity to detect visceral signals, such as those arising from the lungs, heart, or stomach has recently come to the forefront of psychological and psychiatric research (Khalsa et al., 2018; Khalsa \& Lapidus, 2016). A critical objective of this work is to determine the mechanisms by which interoception interacts with cognition and emotion, in order to ultimately derive sensitive and specific neuropsychiatric biomarkers from individual indices of visceral sensitivity. The majority of studies along these lines attempt to measure "interoceptive accuracy" (iACC) in the cardiac domain, as measured by the Heartbeat Counting (HBC) task (Dale \& Anderson, 1978; Schandry, 1981), and similar heartbeat tracking or tapping tasks (Flynn \& Clemens, 1988). While easy to implement, these tasks suffer from serious methodological challenges that obscure their interpretation. To overcome these challenges, we developed a novel psychophysical approach to measure the accuracy, bias, and precision of interoceptive beliefs in the cardiac domain.

The measurement of interoceptive accuracy presents a unique challenge compared to that of exteroception: unlike vision or touch, the heart is not typically amenable to direct experimental control. The inability to control the information present in the stimulus (e.g., the heartbeat) places hard constraints on interoception research, such that most extant tasks ask participants to count uncontrolled endogenous states (e.g., heartbeats) or to determine whether exteroceptive stimuli are synchronized with said states. While these tasks are widely used, they suffer from a number of confounds which place strong limitations on their reliability, interpretability, and validity (for review see Brener \& Ring, 2016; Desmedt et al., 2018; Desmedt, Corneille, et al., 2020; Ring \& Brener, 2018; Zamariola et al., 2018).

A central issue associated with the use of the HBC or similar tasks concerns the role of subjective beliefs about one's own heart rate. These simple measures require participants to silently attend to and count their heartbeats for various intervals, or to tap in rhythm to felt beats. Several authors point out that participants could exploit various strategies to increase 
their accuracy (Clemens, 1979; Flynn \& Clemens, 1988; Pennebaker \& Hoover, 1984). Crucially, even when the heart rate is directly modulated by as much as 100 beats per minute (BPM) via pacemaker, counted heartbeats showed little alteration beyond expectations about different sitting or standing postures on the heart rate (Windmann et al., 1999). Accordingly, participants' subjective prior beliefs about the heart rate have been repeatedly found to be more predictive of counts than actual heartbeats (Ring \& Brener, 1996), and these beliefs can be manipulated via false feedback independently of any true change in heart rate (Ring et al., 2015). A more accurate prior knowledge about one's own heart rate, e.g. amongst medical practitioners or athletes, can influence HBC accuracy scores (Murphy et al., 2018), such that when explicitly instructing participants not to estimate beats, but to instead count felt ones, this bias is reduced (but see Desmedt et al., 2018). More recently, the validity of the HBC task has been further questioned by reports showing that interoceptive accuracy scores are largely driven by under-counting (Zamariola et al., 2018), suggesting that HBC-derived scores are merely a rough reflection of subjective beliefs about the heart rate (Desmedt, Luminet, et al., 2020).

These reports raise serious concerns given the rising interest in interoceptive measurements as potential psychiatric biomarkers (Eggart et al., 2019; Forkmann et al., 2019; Paulus \& Stein, 2010). This poor construct validity could also explain why little to no relationship between $\mathrm{HBC}$-derived scores and various psychiatric symptom measures has been found at the meta-analytic level (Desmedt, Houte, et al., 2020). Here, we argue that the inconsistencies between these $\mathrm{HBC}$-derived scores and interoceptive ability could be better handled by more rigorous measurement and modelling of the role of subjective beliefs in cardiac interoception. Although these tasks were originally designed to be objective and selective measures of the ability to detect afferent cardiac sensory information, they fail to account for factors confounding score variance, such as prior beliefs about the heart rate and other common introspective or self-report biases. In particular, these approaches struggle to dissociate interoceptive sensitivity, bias, and accuracy, confounding the role of subjective vs. objective performance in interoceptive measures.

Another commonly used task, the Heartbeat Discrimination (HBD) task (Whitehead et al., 1977), suffers from different, but similarly serious drawbacks. This method presents participants with a tone series whose onset times are delayed at different intervals relative to the R-wave. Tones presented approximately at systole (typically, $\mathrm{R}+170 \mathrm{~ms}$ ) are treated in 
signal theoretic terms as the "signal plus", while tones presented at a variable time after systole (typically, $\mathrm{R}+300 \mathrm{~ms}$ ) are treated as "signal minus". This design is based on strong assumptions about when, relative to the cardiac phase, participants are most likely to feel the heartbeat. These assumptions have been challenged by results obtained using a similar task based on a method of constant stimuli (MCS), where tones are presented at 5 different intervals with respect to the R-wave. This latter task showed that individuals vary substantially in when they perceive a heartbeat (Brener et al., 1993; Brener Jasper \& Ring Christopher, 2016; for review see Ring \& Brener, 2018), undermining the notion that the HBD can be used as a signal theoretic measure to delineate sensitivity and bias. However, while the MCS likely improves the quantification of single-beat detection when compared to the HBD, both tasks require participants to simultaneously attend to an exteroceptive and interoceptive multi-sensory input, a difficult cognitive task which obscures the relationship to interoception. Moreover, the MCS requires long testing times (as much as 1 hour), which can be problematic for clinical populations, and further requires sophisticated equipment capable of achieving precise cardiac-tone synchrony and stimulus time. As such, there is a need for robust, accessible measures that are amenable to clinical settings, and which can flexibly dissociate the bias, sensitivity, and precision of cardioceptive decisions.

More broadly speaking, we also question the utility of restricting cardioceptive measurements to afferent signal sensitivity only, as increasingly influential "interoceptive predictive processing" models emphasize the top-down, belief-driven nature of embodied perception. On these accounts, subjective interoceptive sensations are more likely to reflect the integration of top-down, prior expectations about the bodily self with ascending sensory inputs, with each signal weighted by their respective precision or confidence (Allen, 2020; Allen \& Friston, 2018; Barrett \& Simmons, 2015; Seth, 2013). The embodied organism is thus a multisensory agent which integrates interoceptive and exteroceptive sensory information to create a unified perception of self. From a phenomenological perspective, the detection of pure visceral-cardiac afferents is an extremely rare and odd phenomenon, probably uncommon to how we typically experience cardiac sensations. It could be argued that cardiac sensations are instead perceived in a diffuse, non-specific fashion, together with tactile (e.g., driven by arterial pulsation against the skin) or auditory sensations.

We argue then that the measurement of subjective beliefs regarding the heart rate, and their integration with ongoing bodily and arousal dynamics, is a core aim for interoception 
research and the search for associated clinical biomarkers. We further put forth that an aberrant and persistent cardiac belief (e.g., that one's heart is racing) is likely to be a more clinically relevant and ecologically valid measure than the ability to specifically detect when in time a single beat occurs.

To measure these beliefs in a sensitive and specific fashion, we developed the heart rate discrimination task (HRD), a novel psychophysical Bayesian approach to estimate the accuracy, bias, and precision of cardiac beliefs. This psychophysical approach measures the explicit perceptual belief about how fast one's heart is currently beating, as well as the "precision" or uncertainty around this point. By presenting stimuli dynamically across trials, titrated to the current heart rate, we can estimate psychometric perceptual and metacognitive curves indicating participants' ability to update cardiac beliefs under different conditional manipulations. This has multiple core benefits beyond mere self-report, as it enables us to simultaneously assess interoceptive accuracy and precision using explicit behavioural responses. This then enables to both distinguish between interoceptive sensitivity and bias, and also to combine trial by trial manipulations of, for example, prior expectations, bodily arousal, or other contextual manipulations with these measures to tease apart the sources of information that shape these beliefs.

To demonstrate the utility of the HRD for measuring cardiac beliefs, characterize the overall heart rate psychometric function, and to establish the face validity of this approach, we measured HRD performance in 215 participants at a resting heart rate while seated upright in a standard testing booth, together with heartbeat counting scores. To further establish internal (test-retest) reliability, we re-tested HRD performance in the same participants following a 6-week period. Our results demonstrate that cardioceptive beliefs are reliably and robustly measured by the task across both sessions. Further, we find that cardioceptive beliefs are more negatively biased, imprecise, and associated with poorer metacognitive insight relative to a matched exteroceptive control condition. 


\section{Methods}

\section{Heart Rate Discrimination task}

\section{Task Overview}

To measure the bias, accuracy, precision, and metacognitive calibration of interoceptive beliefs, we created the Heart Rate Discrimination task (HRD) (See Fig. 1). The task asks participants to first attend to their own cardiac sensations and to then to decide on each trial whether a 'feedback' tone series is faster or slower than their own heart rate in a 2-Interval Forced Choice design (2-IFC). To control for possible non-interoceptive processes such as working memory or general temporal estimation bias, we also implemented a carefully matched exteroceptive control condition, in which participants had to discriminate whether a series of tones was faster or slower than another 'reference' sequence of tones.

a. Heart Rate Discrimination task
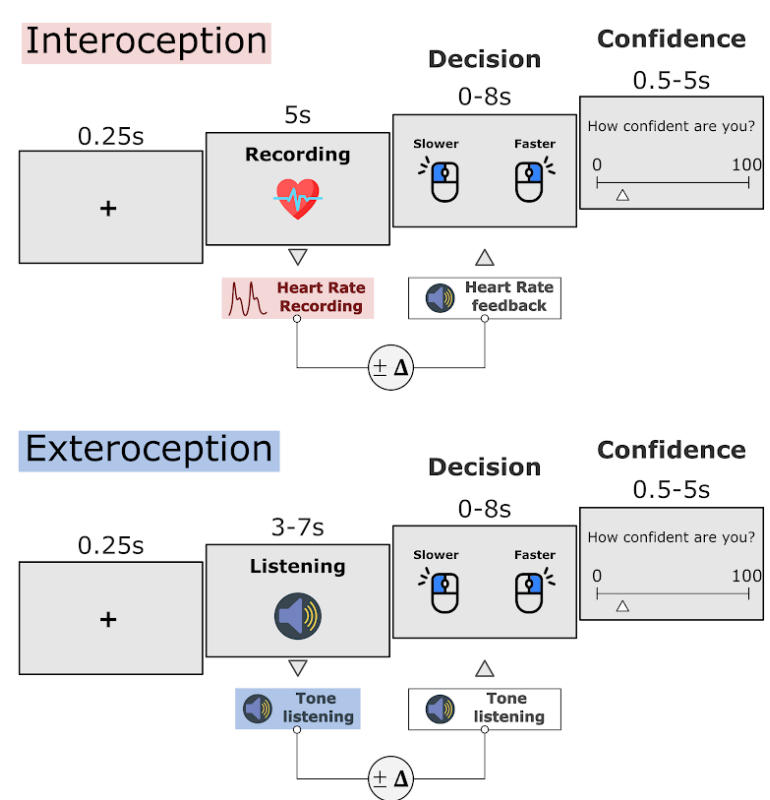

b. Slope and threshold estimates of interoceptive and exteroceptive psychometric functions
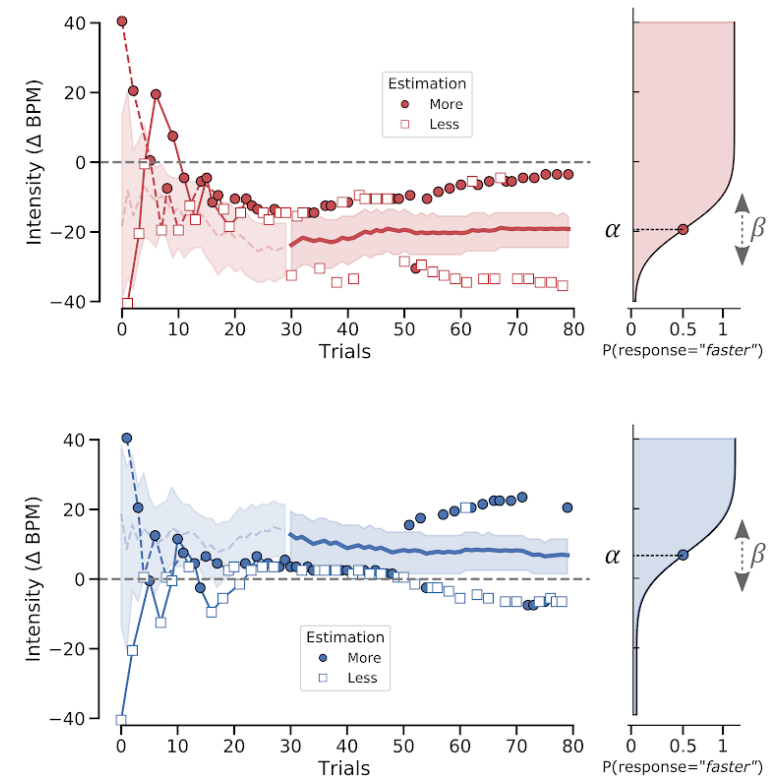

Legend Figure 1: A. Heart Rate Discrimination Trial Design (Session 1). Participants were presented with 160 trials testing their exteroceptive (blue) and interoceptive (red) accuracy and precision (80 in each condition in randomised interleaved order). During interoceptive trials, participants were instructed to attend to their own heart rate for 5 seconds, while it was recorded using a pulse oximeter. The average heart rate for the trial was then computed and used to select the frequency of the tones presented during the decision phase, increased or decreased by an intensity value generated by the staircase, i.e. $\Delta$-BPM. During exteroceptive trials, a sequence 
of tones was presented to the participant with a frequency randomly chosen between 40 and 100 BPM. This frequency was then used to select the beeping frequency of the tones presented during the decision phase, increased or decreased by a value generated by the staircase procedure. $\Delta$-BPM values were controlled by separate staircases for each condition. To estimate metacognitive ability for each modality, at the end of each trial, participants were asked to rate their subjective decision confidence (from 0 - guess to 100 - certain). B. Staircases for each condition from an exemplary subject. Trials rated as faster or slower are depicted with circles or squares respectively. The shaded area represents the $95 \%$ CI of the threshold posterior distribution. On the right panel, the resulting cumulative normal distribution is plotted using the final parameters estimated by the Psi procedure.

To estimate psychometric functions for both conditions, we applied a well-established adaptive Bayesian psychophysical method ("psi”) (Kingdom \& Prins, 2016; Prins \& Kingdom, 2018). This technique adaptively estimates the probability of a participant responding that the feedback tones were "faster" or "slower" than the true heart rate (interoception), or the reference tone (exteroception) on each trial, given the frequency difference between the two stimuli, or $\Delta$-Beats Per Minute $(\Delta-B P M)$. This procedure estimates the point of subjective equality (PSE) both for interoceptive and exteroceptive decision processes in the same $\Delta$-BPM units. The PSE is henceforth referred to as the threshold of the psychometric function. This threshold represents the difference between the true frequency of the heart rate and the estimated cardiac frequency by the participant (see Fig 1. the threshold is denoted $\boldsymbol{\alpha}$ ), such that its absolute value can be taken as a measure of accuracy and its sign plus magnitude as a measure of bias. A negative threshold, therefore, indicates the degree to which a participant underestimates their cardiac frequency, while a positive threshold indicates an overestimation. In addition to the threshold measure, the procedure further estimates the slope of the psychometric function (denoted $\boldsymbol{\beta}$ in Fig 1.), which represents the precision, or uncertainty, around this decision threshold, also in units of $\Delta$-BPM. A larger, more flattened slope value reflects a more uncertain, or imprecise decision process.

This approach to estimating the trial-by-trial psychometric function has several methodological benefits. While many existing interoception tasks involve coarse-grained responses collected over few trials, the adaptive, psychophysical nature of our task enables us to model the relationship between the heart rate, subjective belief, and metacognitive confidence on a trial-by-trial basis. In this way, the HRD can control for a variety of 
low-level confounds such as attentiveness or individual differences in baseline heart rate. Further, it opens the possibility of computational modelling of the interoceptive process using, for example, ballistic evidence accumulation or probabilistic Bayesian models to capture how sensory evidence and/or prior beliefs might influence the decision process (Mathys et al., 2014; Wiecki et al., 2013). As each trial lasts approximately 15 seconds, and the staircase converges on a near-threshold value after as few as 10-20 trials, the task is able to collect as many as 80 near-threshold trials in just 25 minutes of testing. This improves its amenability for estimating metacognitive awareness and other decision variables, particularly, in clinical populations where testing time may be limited due to various practical constraints.

\section{HRD Trial Design}

During interoceptive trials, participants silently attend to their heart rate for 5 seconds, e.g., in a "heart listening" phase (see Fig.1), during which the heart rate is monitored using a soft-clip pulse oximeter placed on one of the fingers of the non-dominant hand. The raw signal is analyzed in real-time using a systolic peak detection algorithm, and the heart rate calculated as the average of the inter-pulse interval (see Physiological Analyses, below). After this listening phase another sequence of five auditory tones was presented (frequency: $440 \mathrm{~Hz}$; duration: $200 \mathrm{~ms}$ ). At any point during the auditory feedback, the participant could press the right or left mouse button to indicate whether the feedback sequence was faster or slower than their estimated average heart rate, terminating the response and feedback period. The maximum response time for the type 1 decision task was 8 seconds. Following the decision interval, participants provide a subject confidence rating (from 0 - uncertain to 100 certain, minimum possible response time: 0.5 seconds; maximum: 5 seconds), and the next trial begins. To prevent motor preparation of the confidence rating, the starting point of the rating scale cursor is randomly jittered around the midpoint by $+/-15 \%$ of the scale length.

Crucially, the frequency of the second tones was adjusted with respect to the frequency of the first tones (exteroceptive modality) or the recorded cardiac frequency (interoceptive modality). This difference is denoted $\Delta$-BPM and corresponds to the stimulus intensity manipulated by the staircases (see the Staircase procedure section below). For example, if the heart rate recorded during the listening condition is $60 \mathrm{BPM}$, and the $\Delta$-BPM value is -15 , the feedback tone frequency will be set to 45 BPM. In this example, if the participant answers "Slower", this is considered a correct answer, otherwise, this is considered an incorrect 
answer. In this way, the staircase procedure hones in on the point of subjective equality, or threshold $(\boldsymbol{\alpha})$, at which the participant is equally likely to respond "Faster" or "Slower".

During exteroceptive trials, participants compared two sequences of tones, instead of comparing their own heart rate with the feedback tone sequence. Here, the first ('reference') tone sequence frequency was randomly selected from a uniform distribution (lower bound = 40 BPM, upper bound = 100 BPM, signal frequency: $440 \mathrm{~Hz}$; tone duration: $200 \mathrm{~ms}$ ), and the second tone sequence frequency presented at a BPM above or below this value as determined by the staircase procedure. After this listening phase, the participants underwent the same decision and confidence task as in the interoceptive trials, that is, to decide whether the second sequence was faster or slower than the first one. The tone presentation ceased when the response was provided (maximum response time: 8 seconds). As in the interoceptive condition, the intensity $\Delta$-BPM was adjusted across trials using the same adaptive Bayesian approach for estimating threshold and slope.

\section{Adaptive Staircase Procedure}

The primary aim of the HRD task is to estimate the difference between the objective heart rate and the participant's subjective belief about this heart rate (i.e., the threshold $\alpha$ ), as well as the precision or uncertainty around this belief (i.e., the slope $\boldsymbol{\beta}$ ). The HRD achieves this via an adaptive staircase procedure which manipulates the $\Delta$ value that was added to the true BPM to produce the feedback tone frequency. This psychophysical procedure can be described as an appearance-based 2-Interval Forced Choice (2-IFC) similar to the 2-Alternative Forced Choice procedure implemented in the Vernier-alignment task, (Kingdom \& Prins, 2016, Chapter 3.3), where varying the degree of difference between two stimuli allows estimating the threshold $(\boldsymbol{\alpha})$ and the slope $(\boldsymbol{\beta})$ of the underlying decision process. In our implementation, HRD thresholds are adaptively estimated using either a standard n-up/n-down staircase to update $\Delta$-BPM on each trial (Dixon \& Mood, 1948), or using a more complex adaptive method known as Psi (Kontsevich \& Tyler, 1999),

Psi is a Bayesian adaptive psychophysical method that manipulates the $\Delta$-BPM deviation values and estimates the slope and the threshold of the underlying sensory psychometric function (Kingdom \& Prins, 2016; Prins \& Kingdom, 2018). The psychometric function relates the deviation $\Delta$ to the proportion of "Faster" decisions using the following formula: 


$$
\psi(\Delta ; \alpha, \beta, \gamma, \lambda)=\gamma+(1-\gamma-\lambda) F(\Delta ; \alpha, \beta)
$$

in which $\Delta$ refers to stimulus intensity, $\psi$ refers to the proportion of trials rated as faster by the participant, and $\gamma$ and $1-\lambda$ are nuisance parameters corresponding to the lower and upper asymptote, respectively. $\boldsymbol{F}$ is the cumulative normal distribution parameterized using threshold $\boldsymbol{\alpha}$ and slope $\boldsymbol{\beta}$. The parameter $\lambda$ is often referred to as the lapse rate and describes the probability of a stimulus-independent negative response. In appearance-based 2-IFC tasks like this one, the lapse rate determines both $\gamma$ and $1-\lambda$. Here, this parameter was fixed, because it is assumed that responses obtained from this kind of task only contain limited information on the real value of this parameter. Further, the estimation of the lapse rate as a free parameter can potentially introduce bias (Prins, 2012). Here, we assumed that $\gamma=\lambda=0.02$.

Irrespective of the staircase procedure (i.e., n-up/n-down or Psi), it should be noted that the psychometric function was not fitted to the proportion of correct trials given the $\Delta$ intensity, but on the probability of a participant making a 'Faster' response given the $\boldsymbol{\Delta}$ intensity (see Fig. 1b). This procedure is known as an appearance-based staircase and entails that the probability of a participant answering "Faster" given an increasing $\Delta$-BPM value is expected to follow a monotonic psychometric function. However, this is not the case for the probability that the response is correct relative to the ground truth HR. Here, the probability of answering correctly increases with $\triangle$-BPM increments towards either positive or negative infinity and is 0.5 around the threshold. As a consequence, the online estimation of an accuracy-based staircase requires dedicated adaptive methods for non-monotonic psychometric functions (García-Pérez, 2014).

\section{Python Cardioception Package}

We implemented two interoception tasks using Psychopy v3.2.3 (Peirce et al., 2019), the classic Schandry Heartbeat Counting Task (Dale \& Anderson, 1978; Schandry, 1981), and the Heart Rate Discrimination task. The code for the two interoception tasks is made publicly available in the Cardioception Python Package (https://github.com/embodied-computation-group/Cardioception). The package natively supports recording and processing pulse rate activity as recorded by the Nonin 3012LP Xpod USB pulse oximeter together with a Nonin 8000SM 'soft-clip' fingertip sensor 
(https://www.nonin.com/) by interfacing with the Systole python package for pulse oximetry (Legrand \& Allen, 2020).

\section{Participants}

218 participants between the ages 18 and 56 (126 females, 91 males, 1 other, age $=24.89 \pm$ 5.30) participated in the study. They were recruited through the Center of Functionally Integrative Neuroscience (CFIN) SONA system participant pool, local advertisements and flyers, social media, and the aarhusbrain.org website. All measures took place at Aarhus University Hospital, Denmark, and were performed on a computer in a behavioural testing room. All participants had normal or corrected to normal vision, and were of at least average proficiency in both Danish and English. All participants were healthy and did not take psychoactive, psychiatric, or cardiovascular medications. Participants who could potentially be pregnant or were breastfeeding, had MRI contraindications (e.g., claustrophobia), or reported that they were not able to abstain from alcohol and drugs 48 hours prior to study participation, were not included in the study. All participants took part in a larger experiment including multiple brain scans, psychiatric inventories, and other behavioural measures (data not reported here). Participants were invited to complete two separate experimental sessions of the HRD task (henceforth referred to as "Session 1" and "Session 2"), separated by 46.89 days on average $(\min =10 ; \max =97 ; \operatorname{std}=23.87) .218$ participants completed session 1 , while 192 (88\%) completed both sessions. 11 participants were excluded from session 1 due to bad signal quality and/or for not having performed the HRD task correctly. 1 participant was excluded from session 1 and 2 due to a prior psychiatric diagnosis. After exclusion, 206 participants completed Session 1 and 191 participants completed Session 2, while 179 completed both sessions. When analysing the confidence ratings, 1 participant from Session 1 and 1 participant from session 2 were dropped due to insufficient variance (see Analysis). 200 participants completed the HBC task, and 7 of them were removed due to poor physiological signal quality. Participants received monetary compensation for each session (350 DKK). The study was conducted in accordance with the Declaration of Helsinki and was approved by the Region Midtjylland Ethics Committee. 


\section{Physiological recordings}

Physiological signals in both sessions were recorded using the Nonin 3012LP Xpod USB pulse oximeter together with a Nonin 8000SM 'soft-clip' fingertip sensor (https://www.nonin.com/) by interfacing with the "Systole" python package (v0.1.3) for pulse oximetry (Legrand \& Allen, 2020). Previous work reported that the pressure exerted by some pulse oximeters on the surface of the skin could provide sensory feedback to some participants, therefore potentially biasing the estimation of interoceptive accuracy (Murphy et al., 2019). We selected the Nonin Pulse softouch pulse oximeter as Murphy and colleagues demonstrated that this device did not elicit fingertip pulse sensations. We further attempted to mitigate this effect by asking the participants to move the device from the index finger to another fingertip if they felt any such sensory feedback, although we did not record the proportion of participants for which this adjustment was made. Participants were further asked to keep their hand still on the table or on their thighs so as to not introduce heart rate measurement errors, due to movements.

\section{Heartbeat Counting Task}

The Heartbeat Counting (HBC) task is perhaps the most widely used and easily implemented task for measuring interoceptive accuracy (Dale \& Anderson, 1978; Schandry, 1981). However, this procedure has been previously criticized, in part because it could be confounded by beliefs about one's own heart rate (Desmedt, Corneille, et al., 2020). To better validate and interpret our new HRD measure, we implemented a revised version of the HBC (Garfinkel et al., 2015), together with specialized instructions to reduce the role of bias in the derived interoceptive accuracy (iACC) scores. Considering the substantial evidence that HBC scores are influenced by beliefs in the heart rate, we expected to observe significant correlations between HBC iACC scores and individual HRD thresholds. Participants were asked to count their heartbeats for various periods of time while sitting silently. The HBC task consisted of 6 trials and lasted 25, 30,35, 40, 45 or 50 seconds. The order of the trials was randomized across participants. The HBC task was measured only in Session 1. 


\section{HRD Task Procedure - Session 1}

At Session 1, the task comprised 160 trials, equally distributed between the interoceptive and the exteroceptive conditions. For each condition, the first 30 trials were run using an adaptive 1-up-1-down staircase procedure, and the remaining 50 trials were run using a Psi procedure (Kontsevich \& Tyler, 1999). Our intention in combining these two staircase procedures was to ensure that each experiment started with a mixture of trials that would be clearly perceived as "Faster" or "Slower" by the participant. The initial up/down staircases consisted of 2 randomly interleaved 1-up 1-down staircases per condition initialized at high $(\Delta$-BPM $=40)$ and low $(\Delta-\mathrm{BPM}=-40)$ starting values following the recommendations of Cornsweet (Cornsweet, 1962). The psi staircases were initialized at starting values informed by the 1-up 1-down staircases, achieved by updating psi in the background with the intensity values and responses recorded during the first 30 up/down trials. Based on our own pilot studies, psi was initialized such that the prior for $\alpha$ was uniformly distributed between -40.5 and 40.5 and the prior for $\boldsymbol{\beta}$ was uniformly distributed between 0 and 20. The $\boldsymbol{\alpha}$ precision was 1 BPM to ensure that the intensity $\Delta$-BPM $=0$ was excluded a priori and never presented. The $\boldsymbol{\beta}$ precision was set to 0.1 .

The main task was preceded by a tutorial phase, which comprised 5 interoception and 5 exteroception trials with accuracy feedback after the decision and without confidence ratings, as well as 5 interoception trials without feedback, but with confidence ratings, as in the main experiment. This was intended to clarify the instructions and to ensure that participants had an opportunity to practice and adapt to the task prior to beginning the staircase, which can be biased by initial lapses. All auditory tones in Session 1 were presented through the stimulus PC speakers. The total duration of the HRD task was 31.31 minutes on average $(S D=3.32, M I N=24.27, M A X=42.46)$.

\section{HRD Task Procedure - Session 2}

To assess the internal (test-retest) reliability of HRD performance, all participants were invited back for a second testing session. Here, all aspects of the HRD were as in Session 1, with the exceptions detailed below. 
The total duration of the HRD at Session 2 was 22.69 minutes on average $(S D=2.45$, $M I N=18.81, M A X=31.81)$. Due to a change in our testing environment, which exposed participants to additional MRI noise, we opted to deliver auditory stimuli via over the ear headphones to limit external distractions. We also increased the maximum decision time from 5 to 8 seconds. To optimize psychometric estimation, we made the following changes to the parameters and overall adaptive procedure. In particular, we observed a ceiling effect in the Session 1 slope parameters (See Supplementary Fig. 2.b), likely induced by an overly restrictive range on the slope prior distribution. To improve the estimation of this parameter, we increased this range from 0-20 in Session 1, to 0-25 in Session 2. The range of the threshold was also increased from [-40.5, 40.5] in Session 1 to [-50.5, 50.5] in Session 2. We also simplified the staircase procedure in Session 2, running only the Psi staircase instead of the dual staircase approach described earlier. As the 1-up-1-down staircase initialization was intended to ensure participants heard a sufficient number of positive and negative $\Delta$-BPM trials (i.e., trials in which the feedback was truly faster or slower than their own true heart rate), we instead implemented "catch" trials presented at fixed intervals above and below zero $\Delta$-BPM. The catch trial responses were not used to update the Psi staircases, yet ensured that once the staircase had converged, subjects still occasionally received faster or slower ground-truth trials. In Session 2, for each modality (Interoception, Exteroception) we used 12 catch trials, along with 48 Psi trials, for a total of 60 trials. In general, these changes improved the stability and reliability of staircase convergence (see Supplementary Fig 2.e).

\section{Analysis}

\section{Statistical Analysis and Software}

Statistical analyses were conducted using Pingouin v0.3.9 (Vallat, 2018). The Bayes Factors were computed using a Cauchy scale of 0.707 and the p-values for the 2-way repeated measure ANOVAs were adjusted using the Greenhouse-Geisser correction. All correlation coefficients were tested using skipped correlations as implemented in Pingouin, which are robust to outliers (Pernet et al., 2013). Where applicable, outliers were detected and rejected using the absolute deviation around the median rule (Leys et al., 2013). Test-retest reliability was tested using the Pearson correlation from the same package. Figures were created using 
Matplotlib (Hunter, 2007), Seaborn (Waskom et al., 2020) and Arviz (Kumar et al., 2019).

Distributions for repeated measures are represented using an adaptation of raincloud plots (Allen et al., 2021). All preprocessed data and analysis scripts supporting the results described in this paper are available at https://github.com/embodied-computation-group/CardioceptionPaper.

\section{Bayesian Modelling of Psychometric Functions}

Although Psi adaptively estimates slope and threshold parameters at each trial, we elected to apply a post hoc modelling approach to improve psychometric estimation. The post hoc modelling was applied after rejecting trials with extremely fast $(<100 \mathrm{~ms})$ responses or during epochs containing unreliable cardiac signals. In general, this approach yielded highly similar results as the psi estimates - see supplementary materials (Supp Fig 1 \& 2) for a comprehensive analysis. We used the absolute deviation around the median rule (Leys et al., 2013) to identify and reject outliers in the instantaneous heart rate time-series. Trials were rejected if the average of the heart rate was considered an outlier, or if the standard deviation of the pulse-to-pulse intervals was detected as outliers when compared to the other trials. This ensured that we only included responses in which the participant was in principle able to correctly estimate their own cardiac frequency. We implemented Bayesian modelling of the psychometric functions using PyMC3 (Salvatier et al., 2016, p. 3). We used the NUTS sampling algorithm (Hoffman \& Gelman, 2011) to update and estimate the posterior probability of the slope $(\boldsymbol{\beta})$ and threshold $(\boldsymbol{\alpha})$ parameters $\left(\mathrm{n}_{\text {chains }}=2, \mathrm{n}_{\text {tuning }}=4000, \mathrm{n}_{\text {samples }}=1000\right)$ for each subject and modality separately. We used a cumulative normal function so the results can be compared to what is estimated by the Psi staircase (see Supplementary Material Fig. 2). The psychometric model parameters were defined as:

$$
\begin{aligned}
\alpha & \sim \operatorname{Uniform}(-40.5,40.5) \\
\beta & \sim \operatorname{Uniform}(0,40) \\
\theta_{i} & =\Phi\left(x_{i}, \alpha, \beta\right) \\
r_{i} & =\operatorname{Binomial}\left(\theta_{i}, n_{i}\right)
\end{aligned}
$$

Considering the $i$ th intensity levels, for the trials with a stimulus intensity $x_{i}$ we observed a total of $n_{i}$ responses, among which $r_{i}$ were "Faster" responses. Here, $\boldsymbol{\phi}$ is the cumulative normal function defined by: 


$$
\Phi(x, \alpha, \beta)=\frac{1}{2}+\frac{1}{2} * \operatorname{erf}\left(\frac{x-\alpha}{\beta * \sqrt{2}}\right)
$$

Because we aimed to correlate the resulting scores with other variables and were not interested in group-level means, we fitted the model for each subject and each modality separately in a non-hierarchical manner. These post hoc models are included in the cardioception toolkit, and future releases will provide easy to use hierarchical group estimation, to facilitate for example between groups analyses (Valton et al., 2020). All subsequent psychometric behavioural analyses were performed on the post hoc estimated parameters.

\section{Signal Theoretic Modelling of Perceptual and Metacognitive Sensitivity}

For all signal theoretic analyses, one participant was excluded from Session 1 ( $n=204$, after exclusion) and another participant from Session $2(\mathrm{n}=190$, after exclusion) due to invalid confidence ratings (all ratings around the same value). For these analyses, accuracy was coded such that a "Faster" response was correct only when the intensity $\Delta$-BPM was greater than 0 , and a "Slower" response was correct only when the intensity $\Delta$-BPM was smaller than 0. Confidence ratings were binned into 4 equally spaced bins prior to modelling using the discreteRatings() functions from metadPy, a custom python package for metacognition modeling (https://github.com/LegrandNico/metadPy). We used a standard Signal Detection Theory (SDT) approach to estimate type 1 (i.e., perceptual) and type 2 (i.e., metacognitive) bias and sensitivity from the binned confidence ratings. Briefly, this model operationalizes metacognitive "insight" as the sensitivity of subjective confidence ratings to ground truth accuracy; e.g., by defining a receiver-operating characteristic (ROC) curve relating metacognitive "hits" - $p($ Confidence $=$ High $\mid$ Response = Correct $)$ - and "misses" $p($ Confidence $=$ High $\mid$ Response $=$ Incorrect $)$ - (Fleming \& Lau, 2014; Maniscalco $\&$ Lau, 2012a). This measure is known as meta- $d$ ', and is an index of metacognitive sensitivity akin to d'. However, as meta-d is known to be influenced by overall $d$ ', and interoceptive accuracy is generally lower than exteroceptive on our task, we analyzed the parameter M-ratio (meta- $d$ '/d'), also known as "metacognitive efficiency". This parameter operationalizes metacognitive insight in signal theoretic units; e.g., the proportion of available sensory evidence utilized by the subjective confidence response. Perceptual and 
Metacognitive parameters were estimated using an adapted hierarchical Bayesian model from the HMeta-d toolbox (Fleming, 2017). We reparameterized this model to implement a paired-samples t-test estimating the within-subject impact of modality (interoceptive vs. exteroceptive) on M-ratio. The significance of this effect was then assessed by checking if the $94 \%$ highest density interval $\left(\mathrm{HDI}_{94 \%}\right)$ includes zero or not.

\section{Physiological Analysis}

The time-series recorded through photoplethysmography (PPG) were analysed using Systole v0.1.3 (Legrand \& Allen, 2020). The PPG signal, sampled at $75 \mathrm{~Hz}$, is a measure of peripheral blood oxygenation level, in which cardiac cycles can be tracked by detecting abrupt increases following cardiac contraction and blood circulation (i.e., systolic peaks). The signal was first resampled to $1000 \mathrm{~Hz}$ using linear interpolation. This procedure simplifies the measurement of the pulse-to-pulse intervals and can refine the peak detection precision, and the resulting heart rate when the initial sampling rate is low (Quintana et al., 2016). Clipping artefacts were removed using cubic spline interpolation (van Gent et al., 2019), the signal was then squared for peak enhancement and normalized using the mean + standard deviation using a rolling window (window size: 0.75 seconds). All positive peaks were labeled as systolic (minimum distance: 0.2 seconds). This procedure was applied both for the online heart rate recording during the Heart Rate Discrimination task (segments of 5 seconds) and for the Heartbeat Counting task. If any interbeat interval higher than 120 BPM or lower than 40 BPM was detected during the online recording of the Heart Rate Discrimination task, an error message was presented on the screen to ask the participant to stay still, and the trial was started again, up to 5 times consecutively before dropping the trial. As the correct detection of heartbeats is critical for the Heartbeat Counting task, we ran additional artefacts correction steps to control for erroneous or missed detection of some heartbeats. Extra heartbeats (i.e., erroneous labeling of peaks in PPG signal) were automatically removed using an artefact correction algorithm (Lipponen \& Tarvainen, 2019) implemented in Systole (Legrand \& Allen, 2020). All raw time-series were manually inspected to ensure correct systolic peak detection. The HTML reports detailing these preprocessing steps of the HRD and the HBC tasks are made available online with the GitHub Repository associated with this paper. 


\section{Heartbeat Counting Analysis}

We derived an accuracy score following previous recommendations (Garfinkel et al., 2015; Hart et al., 2013) as follows:

$$
\text { Score }=1-\frac{\left|N_{\text {real }}-N_{\text {reported }}\right|}{\frac{N_{\text {real }}+N_{\text {reported }}}{2}}
$$

This score has a maximum at 1 and indicates the similarity between the objective recorded number of heartbeats and the number reported by the participant (a score of 1 indicating a perfect match). We used the absolute deviation around the median rule (Leys et al., 2013) to automatically detect and remove extreme responses that are more likely to reflect erroneous numbers provided by the participant. The remaining scores were subsequently averaged for each participant.

\section{Results}

\section{Characterizing the Interoceptive and Exteroceptive Psychometric Function}

We first analyzed the threshold $(\boldsymbol{\alpha})$ and slope $(\boldsymbol{\beta})$ psychometric parameters using the estimates from the post hoc Bayesian model, separately for both sessions (see Methods for more details and Supplementary Results for comparison of psi and post hoc parameters). These analyses serve to both characterize the overall shape of the two psychometric functions, which can inform the setting of prior parameters in future experiments, and assess how belief accuracy, bias, and precision differed between the two conditions. Further, to explore possible Session by Modality interactions, we fit repeated measures ANOVAs to each parameter of interest.

A paired sample t-test at Session 1 revealed that threshold was significantly lower in the interoceptive condition than in the exteroceptive condition ( $\operatorname{mean}_{\text {Intero }}=-6.97, \mathrm{CI}_{95 \%}[-8.59$, -5.37], mean $_{\text {Extero }}=1.36, \mathrm{CI}_{95 \%}[0.9,1.85], t_{(205)}=-9.89, p<0.001, \mathrm{BF}_{10}=1.15 \mathrm{e}+16, d=$ -0.93), indicating a robust negative bias; i.e., heart rate underestimation. This effect was replicated at Session $2\left(\operatorname{mean}_{\text {Intero }}=-8.50,95 \% \mathrm{CI}_{95 \%}[-10.09,-6.91]\right.$, mean $_{\text {Extero }}=0.008,95 \%$ CI $\left.[-0.48,0.50], t_{(190)}=-11.15, p<0.001, \mathrm{BF}_{10}=2.85 \mathrm{e}+19, d=-1.03\right)$. We further noted a marked increase in inter-subject variance for interoceptive thresholds, range Intero $=[-38.3$, 34.0] vs. exteroceptive thresholds, range $_{\text {Extero }}=[-9.8,16.39]$, indicating substantially more 
inter-individual variance in the magnitude of interoceptive biases. In contrast, when compared to a null hypothesis of 0 bias, follow-up one-sample t-tests on exteroceptive thresholds revealed a slight but highly significant positive bias at Session 1 (mean $=1.39, \mathrm{CI}_{95}=[0.9$, 1.89], $\left.t_{(203)}=5.58, p<0.001, \mathrm{BF}_{10}=1.28 \mathrm{e}+05, d=0.99\right)$, which was not present at Session 2 (mean $\left.=0.01, \mathrm{CI}_{95}=[-0.48,0.52], t_{(189)}=0.06, p=0.94, \mathrm{BF}_{10}=0.08, d=0.05\right)$. Finally, exploratory repeated measures ANOVA revealed significant main effects of Session $\left(\mathrm{F}_{(1,178)}=\right.$ $\left.13.20, \eta_{\mathrm{p}} 2=0.06, p<0.001\right)$ and Modality $\left(\mathrm{F}_{(1,178)}=127.53, \eta_{\mathrm{p}} 2=0.41, p<0.001\right)$, indicating that thresholds were significantly reduced across sessions for both modalities, and that interoception was more biased across both sessions, but with no Session by Modality interaction $\left(\mathrm{F}_{(1,178)}=0.60, \eta_{\mathrm{p}} 2=0.003, p=0.43\right)$. See Figures 2 and $6 \mathbf{B}$ for illustration of these effects. Collectively these results show that interoceptive heart rate beliefs are robustly biased towards underestimation, and show greater inter-individual variance, than our matched exteroceptive control condition.

We next consider the slope of the interoceptive and exteroceptive functions. While the threshold indicates the overall accuracy and bias of the decision-making process, the slope characterizes the precision or uncertainty of this process. A higher slope indicates a less steep (i.e., more shallow) psychometric function, indicating lower precision (higher uncertainty) for that condition. A paired sample t-test revealed that slope was significantly higher in the interoceptive condition as compared to the exteroceptive $\left(\operatorname{mean}_{\text {Intero }}=15.34, \mathrm{CI}_{95 \%}[14.39\right.$, $16.36]$, mean $_{\text {Extero }}=9.58, \mathrm{CI}_{95 \%}[8.89,10.39], t_{(205)}=9.05, p<0.001, \mathrm{BF}_{10}=4.97 \mathrm{e}+13, d=$ -0.88). This effect was reproduced in Session 2, with interoceptive slope again greater than exteroceptive slope at retest $\left(\operatorname{mean}_{\text {Intero }}=11.96,95 \% \mathrm{CI}[11.17,12.76]\right.$, mean $_{\text {Extero }}=8.69,95 \%$ CI $\left.[8.11,9.26], t_{(190)}=7.29, p<0.001, \mathrm{BF}_{10}=9.12 \mathrm{e}+08, d=0.67\right)$. Exploratory repeated measures ANOVA further revealed main effects of Session $\left(\mathrm{F}_{(1,178)}=31.27, \eta_{\mathrm{p}} 2=0.14, p<\right.$ $0.001)$ and Modality $\left(\mathrm{F}_{(1,178)}=106.29, \eta_{\mathrm{p}} 2=0.37, p<0.001\right)$, as well as an interaction between these two factors $\left(\mathrm{F}_{(1,178)}=9.46, \eta_{\mathrm{p}} 2=0.05, p=0.002\right)$. Thus, interoceptive slope showed a greater reduction across sessions $\left(t_{(178)}=-5.35, p<0.001, \mathrm{BF}_{10}=4.13+04, d=\right.$ -0.53) than exteroceptive slope $\left(t_{(178)}=-5.35, p<0.001, \mathrm{BF}_{10}=4.13+04, d=-0.53\right)$. Collectively, these results demonstrate that interoceptive beliefs are less precise than exteroceptive. Further, interoceptive precision may be more sensitive to practice and training effects than exteroceptive precision. See Figure 2 and Supplementary Figure 1 for illustration of these effects. 
bioRxiv preprint doi: https://doi.org/10.1101/2021.02.18.431871; this version posted February 18, 2021. The copyright holder for this preprint (which was not certified by peer review) is the author/funder, who has granted bioRxiv a license to display the preprint in perpetuity. It is made available under aCC-BY-NC-ND 4.0 International license.

a. Psychometric parameters
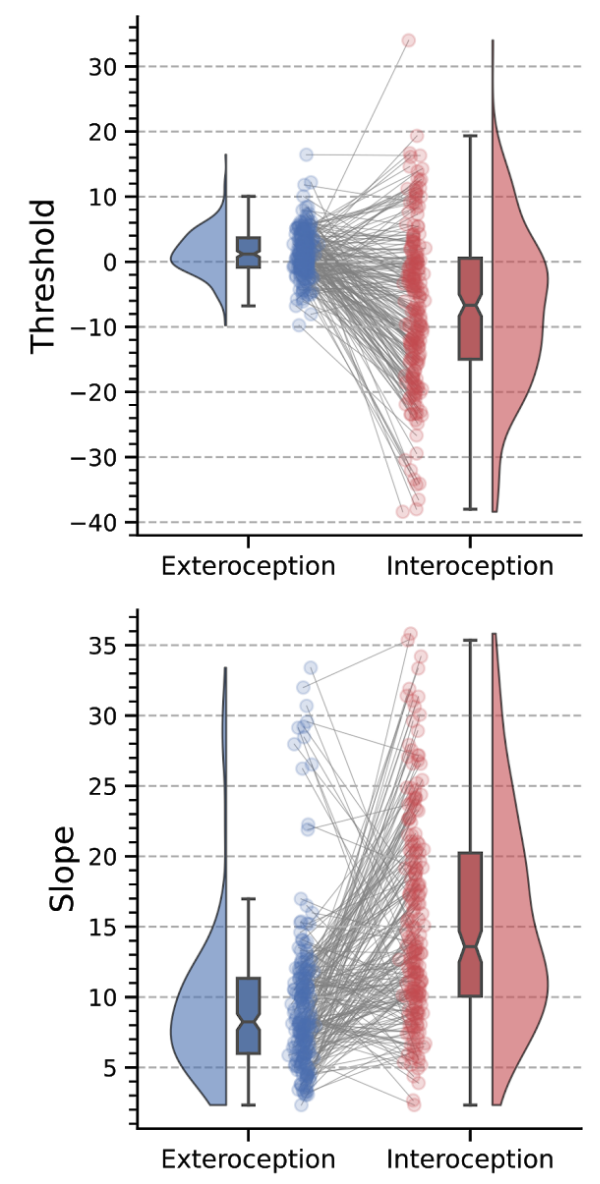

b. Psychometric functions
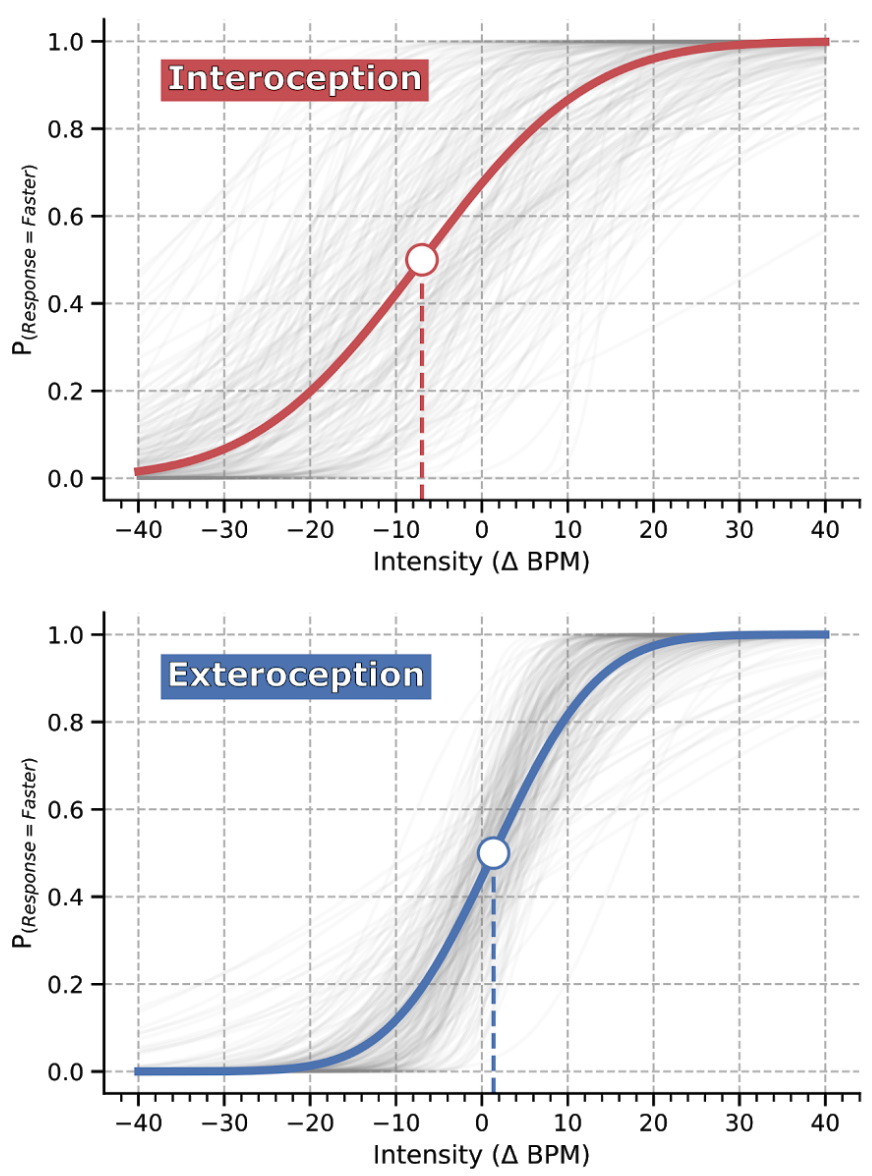

Legend Figure 2: Psychometric parameter estimates and fitted interoception and exteroception psychometric functions (Session 1). A. Repeated measures raincloud plots visualizing threshold and slope parameters of the psychometric functions across the two modalities (interoception and exteroception). Data points for every individual are connected by a grey line to highlight the repeated measure effect. B. The grey lines show individual subject fits. The dark red and blue lines show the grand mean psychometric function, depicting averaged threshold and slope. Grand mean thresholds are marked by the large point, where the psychometric function crosses 0.5 on the ordinate axis. We observed a strong effect of interoception on both slope and threshold as compared to the exteroceptive control condition. The negative bias observed on threshold demonstrates that participants underestimated their heart rate on average. The greater slope indicates a less precise decision process.

\section{Perceptual and Metacognitive Sensitivity}

In addition to the psychometric function underlying the subjective decision process, we also compared objective overall perceptual and metacognitive sensitivity between interoception and exteroception. To assess between condition differences on these indices, we performed 
paired-sample t-tests comparing interoceptive and exteroceptive performance on each key type 1 and type 2 measure (d', average confidence, and m-ratio), as well as exploratory Modality by Session repeated measures ANOVAs on these variables.

The $d$, which reflects discrimination sensitivity, was signifigantly lower in the interoception condition as compared to the exteroception condition during Session 1 $\left(\operatorname{mean}_{\text {Intero }}=1.43, \mathrm{CI}_{95 \%}=[1.34,1.52]\right.$, mean $\left._{\text {Extero }}=2.05, \mathrm{CI}_{95 \%}=[1.96,2.14]\right), t_{(203)}=-9.42, p<$ $\left.0.001, \mathrm{BF}_{10}=5.06 \mathrm{e}+17, d=-0.97\right)$. We replicated this effect in Session $2\left(\operatorname{mean}_{\text {Intero }}=1.87\right.$, $\mathrm{CI}_{95 \%}=[1.79,1.96]$, mean $_{\text {Extero }}=2.25, \mathrm{CI}_{95 \%}=[2.21,2.3], t_{(185)}=-7.98, p<0.001, \mathrm{BF}_{10}=$ $4.45 \mathrm{e}+10, d=-0.77)$. We also performed an exploratory Session by Modality repeated measures ANOVA on these measures to assess overall interactions between these factors. We observed significant effects of both Session $\left(\mathrm{F}_{(1,176)}=59.82, \eta_{\mathrm{p}} 2=0.25, p<0.001\right)$, Modality $\left(\mathrm{F}_{(1,176)}=114.81, \eta_{\mathrm{p}} 2=0.39, p<0.001\right)$, and a Session by Modality interaction $\left(\mathrm{F}_{(1,176)}=7.19\right.$, $\left.\eta_{\mathrm{p}} 2=0.03, p=0.008\right)$. This result shows that across both conditions, sensitivity increased from Session 1 to 2, with the greatest increase being observed in the interoceptive condition.

We next analyzed average subjective confidence, an indicator of metacognitive bias. We found that confidence ratings were significantly lower during the interoception condition as compared to the exteroception condition, both during the first session $\left(\operatorname{mean}_{\text {Intero }}=51.52\right.$, $\mathrm{CI}_{95 \%}=[49.16,53.87]$, mean $_{\text {Extero }}=61.44, \mathrm{CI}_{95 \%}=[59.51,63.57], t_{(203)}=-10.01, p<0.001, \mathrm{BF}_{10}$ $=2.3 \mathrm{e}+16, d=-0.62)$ and the second session $\left(\operatorname{mean}_{\text {Intero }}=57.47, \mathrm{CI}_{95 \%}=[55.41,59.77]\right.$, mean $\left._{\text {Extero }}=64.27, \mathrm{CI}_{95 \%}=[62.4,66.03], t_{(189)}=-7.15, p<0.001, \mathrm{BF}_{10}=4.18 \mathrm{e}+08, d=-0.49\right)$. An exploratory repeated measures ANOVA revealed a main effect of Session $\left(\mathrm{F}_{(1,176)}=26.37\right.$, $\left.\eta_{\mathrm{p}} 2=0.13, p<0.001\right)$, Modality $\left(\mathrm{F}_{(1,176)}=101.37, \eta_{\mathrm{p}} 2=0.36, p<0.001\right)$ and a Session by Modality interaction $\left(\mathrm{F}_{(1,176)}=8.72, \eta_{\mathrm{p}} 2=0.04, p<0.003\right)$. The average confidence was higher in the second session as compared to the first one $\left(t_{(176)}=5.13, p<0.001, \mathrm{BF}_{10}=\right.$ $1.52+04, d=0.30)$, and this increase was larger for the interoceptive condition $\left(t_{(176)}=6.02, p\right.$ $\left.<0.001, \mathrm{BF}_{10}=9.68+05, d=0.36\right)$ than for the exteroceptive condition $\left(t_{(176)}=2.54, p<0.01\right.$, $\left.\mathrm{BF}_{10}=1.93, d=0.18\right)$. Overall, confidence was generally lower for interoceptive vs. exteroceptive confidence.

To assess metacognitive sensitivity for both modalities, we estimated metacognitive efficiency using hierarchical modelling of M-ratio (meta-d'/d') (Fleming \& Lau, 2014; Maniscalco \& Lau, 2012a). We observed that the individual estimated M-ratio values, as estimated by the repeated measure model, were lower during the interoception condition 
$\left(\operatorname{mean}_{\text {Intero }}=0.81, \mathrm{CI}_{95 \%}=[0.78,0.86]\right)$ as compared to the exteroception condition ( $\operatorname{mean}_{\text {Extero }}$ $\left.=0.96, \mathrm{CI}_{95 \%}=[0.92,1.01]\right)$. This tendency is confirmed by inspecting the posterior distribution of the log-transformed repeated measure effect (mean $=-0.19, \mathrm{HDI}_{94 \%}=[-0.36$, -0.06], see Fig 3.c). Because the M-ratio reflects the relation between the amount of evidence for metacognitive judgement and the amount of evidence for the objective decision, our results suggest that $19 \%$ of the interoceptive evidence used for decision in the type 1 task is lost during the metacognitive evaluation of confidence, compared to just $4 \%$ evidence loss for exteroception.

We replicated this finding in Session 2, where interoception M-ratio estimates were again lower $\left(\operatorname{mean}_{\text {Intero }}=0.83, \mathrm{CI}_{95 \%}=[0.8,0.87]\right)$ than those for exteroception $\left(\operatorname{mean}_{\text {Extero }}=\right.$ $\left.0.96, \mathrm{CI}_{95 \%}=[0.92,1.01]\right)$, as well as in the posterior distribution of the repeated measure effect (mean $=-0.17 \mathrm{HDI}_{94 \%}=[-0.26,-0.03]$, see Supplementary Material, Figure3). 


\section{a. Confidence ratings for correct and incorrect responses}
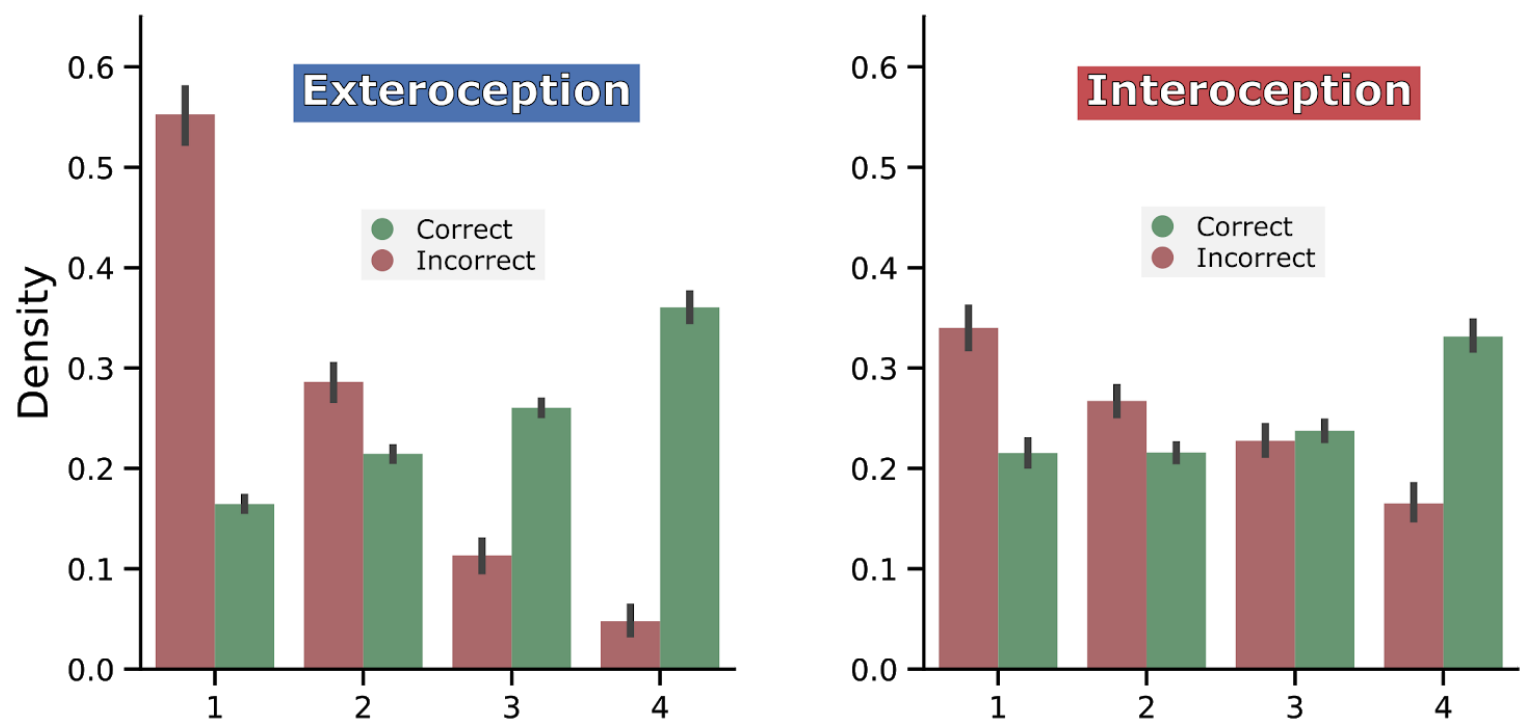

b. Metacognitive efficiency (m-ratio)

c. Effect of interoception on metacognitive efficiency
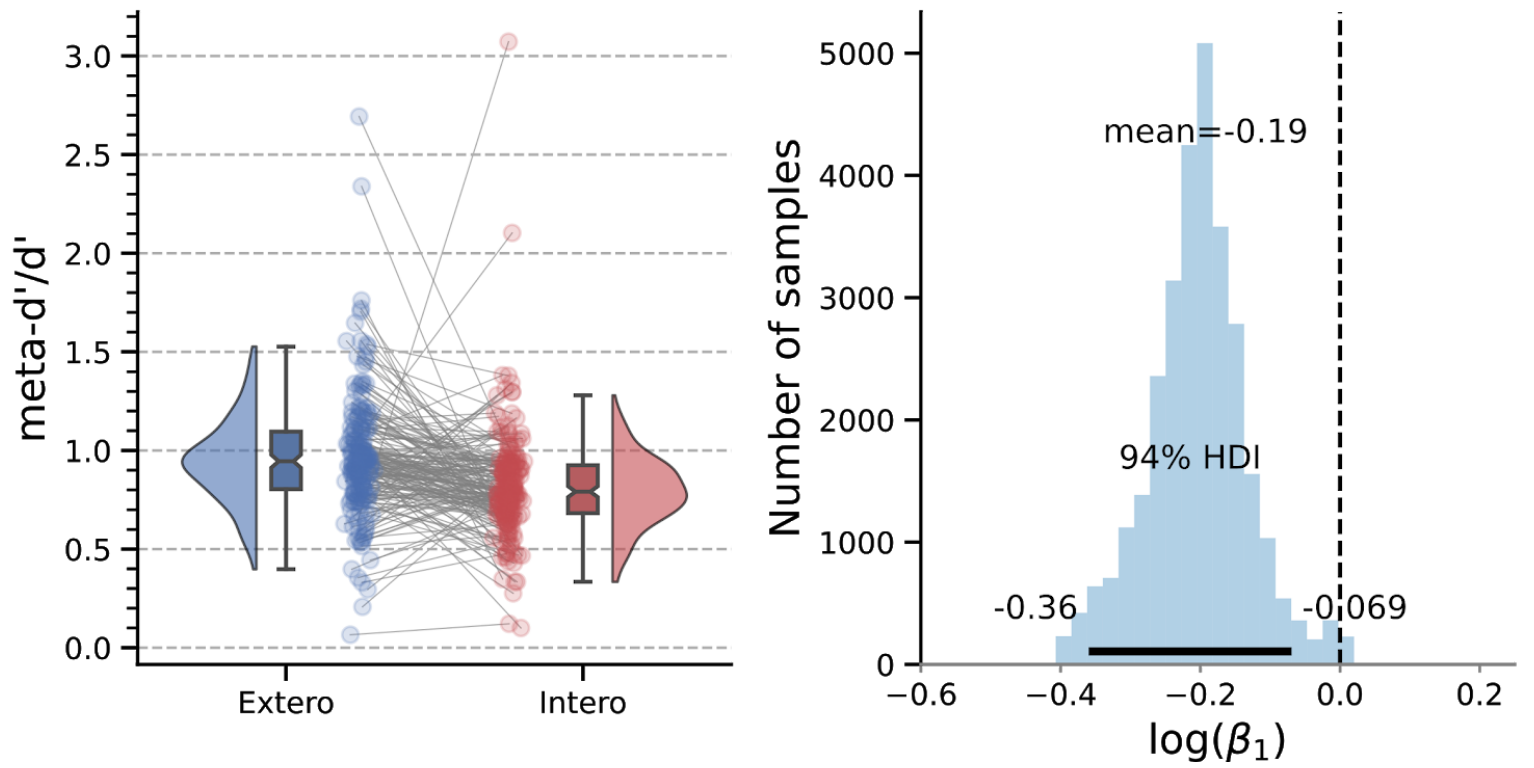

Legend Figure 3: Visualization of metacognitive performance for interoception and exteroception

conditions (Session 1). A. Histogram showing the distribution of binned confidence ratings for correct vs. error trials. Overall, participants were significantly less confident in the interoceptive condition and showed reduced metacognitive sensitivity as indicated by the flattening of the confidence distributions. B. To quantify this effect, we estimated "metacognitive efficiency", a signal theoretic model of introspective accuracy which controls for differences in type 1 (discrimination) performance. Here, an M-ratio of 1 indicates optimal metacognition according to an ideal observer model, whereas values lower than this indicate inefficient use of the available perceptual signal. This model demonstrated that metacognitive efficiency was substantially decreased for interoceptive relative to exteroceptive judgements. C. Histogram of posterior samples from the beta value encoding the difference of interoception-exteroception in the repeated measures hierarchical model. 


\section{Cross-modal Correlations}

To investigate the construct validity of HRD performance measures, we conducted an exploratory correlation analysis relating individual differences in perceptual and metacognitive performance within and between the interoceptive and exteroceptive modalities. For this analysis, we refitted the meta-d' model (Fleming, 2017) separately to each participant (i.e., in a non-hierarchical model), and extracted individual M-ratio values. Here, our aim was to verify whether threshold, slope, or other type 1 or type 2 parameters were correlated across the two conditions. For example, if HRD performance primarily indexed general temporal estimation ability, we would expect a high correlation between interoceptive and exteroceptive thresholds, as well as with other type 1 performance variables. Alternatively, if participants used additional information, such as afferent cardiac sensory information and/or prior beliefs specifically about the heart rate, then we would expect little to no correlation between these parameters. Additionally, previous studies found that interoceptive metacognition is typically uncorrelated to exteroceptive metacognition, suggesting unique inputs for these self-estimates (Garfinkel et al., 2016). However, more recent work suggested the existence of a "metacognitive g-factor" indexed by high inter-modal correlations in metacognitive ability (Mazancieux et al., 2020; Rouault et al., 2018). We, therefore, included both type 1 measures (i.e., threshold, slope, d', response time, and criterion) and type 2 measures (confidence, meta-d', M-ratio) in one exploratory between-subject correlation analysis to probe the degree of within and between modality overlap in parameter estimates. To do so, we performed robust pairwise correlation tests between exteroception and interoception task parameters (Pernet et al., 2013), using a skipped correlation approach and correcting for multiple comparisons using a false-discovery rate $\left(\right.$ FDR, $\left.\mathrm{p}_{\mathrm{FDR}}<0.01\right)$ correction. The resulting Spearman's $\mathrm{r}$ coefficients for Session 1 are summarized in Fig 4.

We observed more robust and consistent correlations between task parameters within each modality (interoception or exteroception), but few significant correlations between task modalities, indicating a high degree of independence between performance on the two task conditions. Interestingly, with the exception of reaction time, type 1 performance was largely uncorrelated between modalities, whereas at the metacognitive level only subjective confidence was highly correlated $\left(\mathrm{r}_{\mathrm{s}}=0.60, \mathrm{CI}_{95 \%}=[0.52,0.69], \mathrm{p}<0.001, \mathrm{n}=204, \mathrm{n}_{\text {outliers }}=\right.$ 
bioRxiv preprint doi: https://doi.org/10.1101/2021.02.18.431871; this version posted February 18, 2021. The copyright holder for this preprint (which was not certified by peer review) is the author/funder, who has granted bioRxiv a license to display the preprint in perpetuity. It is made available under aCC-BY-NC-ND 4.0 International license.

5). These results may suggest that individuals use similar "self-beliefs" about their performance on both task modalities (Fleming \& Daw, 2017). A similar overall pattern was observed in Session 2, albeit with a modest but significant relationship between interoceptive and exteroceptive thresholds $\left(\mathrm{r}_{\mathrm{s}}=0.26, \mathrm{CI}_{95 \%}=[0.13,0.39], p<0.001, \mathrm{n}=190, \mathrm{n}_{\text {outliers }}=6\right.$, see Supplementary Results for the full correlation matrix).

\section{Cross-modal correlations matrix}

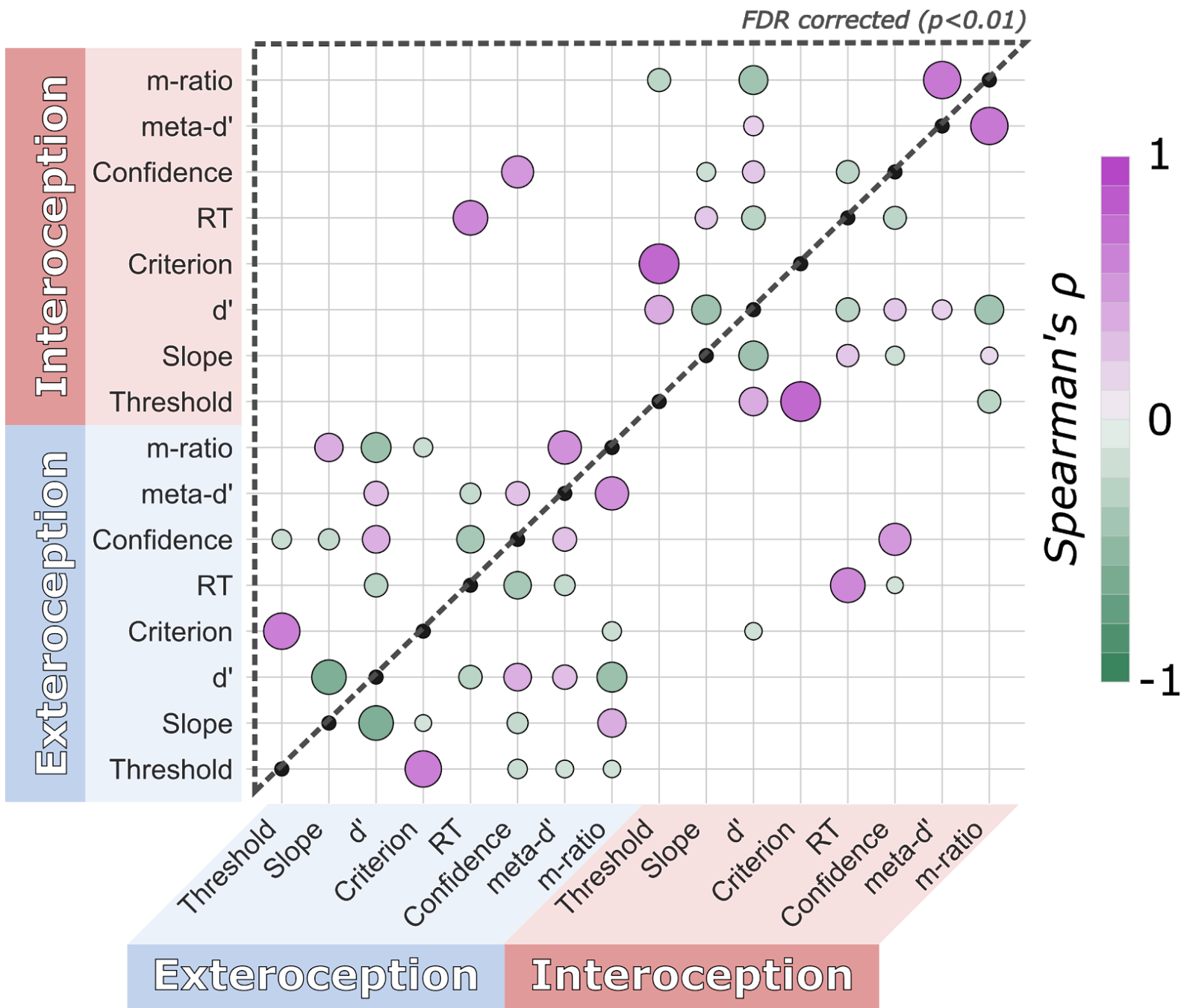

Legend Figure 4: Cross-modal correlation heatmap of task parameters for interoception and exteroception conditions (Session 1). Overall, we observed that behavioural results were correlated within modalities but with limited dependence across modalities, the only exceptions were confidence and response time (RT). Only significant skipped Spearman correlations are represented. The upper triangle only shows results surviving FDR correction $\left(\mathrm{p}_{\mathrm{FDR}}<0.01\right)$, while the lower right triangle of the matrix shows the uncorrected comparisons. Colour and size of individual points indicate the sign and strength of estimated correlation coefficients. See supplementary Fig 4 for Session 2 cross-correlations. 


\section{Correlation with the heartbeat counting task parameters}

As a final check of construct validity, we assessed how our new task relates to the standard heartbeat counting task. We thus correlated HRD performance variables (psychometric thresholds and slopes) with the HBC scores. We found that the interoceptive thresholds from Session 1 were positively correlated with the global heartbeat counting score (see Fig 5.a; $r_{s}=$ $\left.0.29, \mathrm{CI}_{95 \%}=[0.16,0.42], p<0.001, \mathrm{n}=193, \mathrm{n}_{\text {outliers }}=1\right)$. No significant correlation was found between the heartbeat counting score and the exteroceptive threshold $\left(\mathrm{r}_{\mathrm{s}}=-0.04, \mathrm{CI}_{95 \%}=\right.$ $\left.[-0.18,0.1], p=0.58, \mathrm{n}=193, \mathrm{n}_{\text {outliers }}=13\right)$. We further replicated the correlation between HRD threshold and HBC iACC scores in Session $2\left(\mathrm{r}_{\mathrm{s}}=0.19, \mathrm{CI}_{95 \%}=[0.05,0.33], p=0.01, \mathrm{n}\right.$ $\left.=178, \mathrm{n}_{\text {outliers }}=0\right)$.

\section{Correlation with the heartbeat counting task parameters}
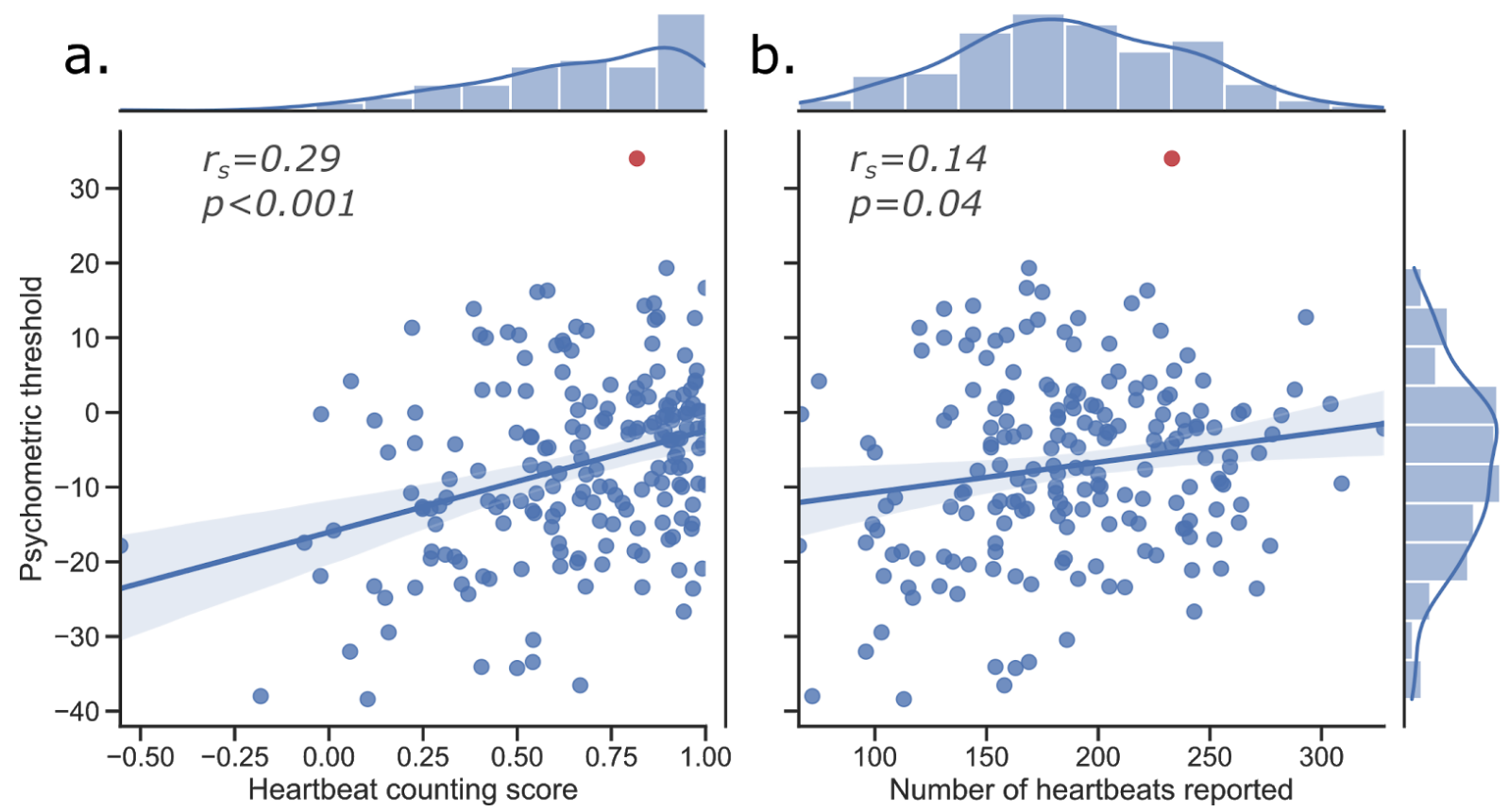

Legend Figure 5: Correlation between the psychometric threshold and heartbeat counting performance.

(Session 1) A. We found that heart rate discrimination (HRD) thresholds correlate positively with heartbeat counting (HBC) interoceptive accuracy scores. A lower threshold (i.e., a more negative bias) on the HRD task was associated with lower performance on heartbeat counting. We suggest that low scores on the heartbeat counting task are associated with a tendency to undercount the number of heartbeats. B. The psychometric threshold was associated with the total number of heartbeats reported during the heartbeat counting task. The correlation was also found while controlling for the heart rate during the task (not shown). These results suggest that participants' inability to reliably count their heartbeats is partially explained by lower interoceptive 
thresholds. Outliers detected by the skipped correlation are reported in red. The $r_{s}$ and $p$ values are from the bootstrapped Spearman coefficient. The regression line is only fitted to non-outlier data points. The shaded area represents the bootstrapped confidence interval $(95 \%)$.

The previous results suggest that the bias observed in the heartbeat counting task might be at least partially explained by the participants' tendency to underestimate their own heart rate. To corroborate this notion, we wanted to verify the association between the psychometric threshold obtained during the HRD task, which quantifies the heart rate underestimation, and the total number of heartbeats reported by the participant during the HBC task (see Fig 5.b). The psychometric threshold was positively correlated with the total number of heartbeats counted by the participants $\left(\mathrm{r}_{\mathrm{s}}=0.14, \mathrm{CI}_{95 \%}=[0.01,0.28], p=0.04, \mathrm{n}=193, \mathrm{n}_{\text {outliers }}=1\right)$. It could be argued here that the actual heart rate of the participant may directly influence the total number of counted heartbeats, as the number of heartbeats that can be potentially counted naturally increases with increments in heart rate frequency. To control for this possible confound, we performed a semi-partial correlation between the psychometric threshold and the total number of counted heartbeats while controlling for the relation between the number of counted heartbeats and the number of actual heartbeats detected in the PPG signal. This analysis revealed a positive correlation between these two variables $\left(r_{\mathrm{s}}=\right.$ $\left.0.20, \mathrm{CI}_{95 \%}=[0.07,0.34], p=0.004, \mathrm{n}=193, \mathrm{n}_{\text {outliers }}=2\right)$.

\section{Reliability of psychometric parameters}

Intrinsic or test-retest reliability is a critical feature of any measurement, in particular, if it is to be useful for clinical diagnostic or intervention purposes. To evaluate reliability, we calculated the correlation coefficient for Session 1 and 2 interoceptive thresholds and slopes, obtained on average 46.79 days apart from each other. Threshold was highly correlated between sessions, showing good reliability $\left(\mathrm{r}=0.51, p<0.001, \mathrm{BF}_{10}=5.04 \mathrm{e}+10\right.$, see Fig 6). In contrast, Slope was not correlated across sessions $\left(\mathrm{r}=0.10, p=0.15, \mathrm{BF}_{10}=0.25\right)$, potentially indicating a poor reliability of this parameter. 
a. Test re-test reliability of the psychometric threshold

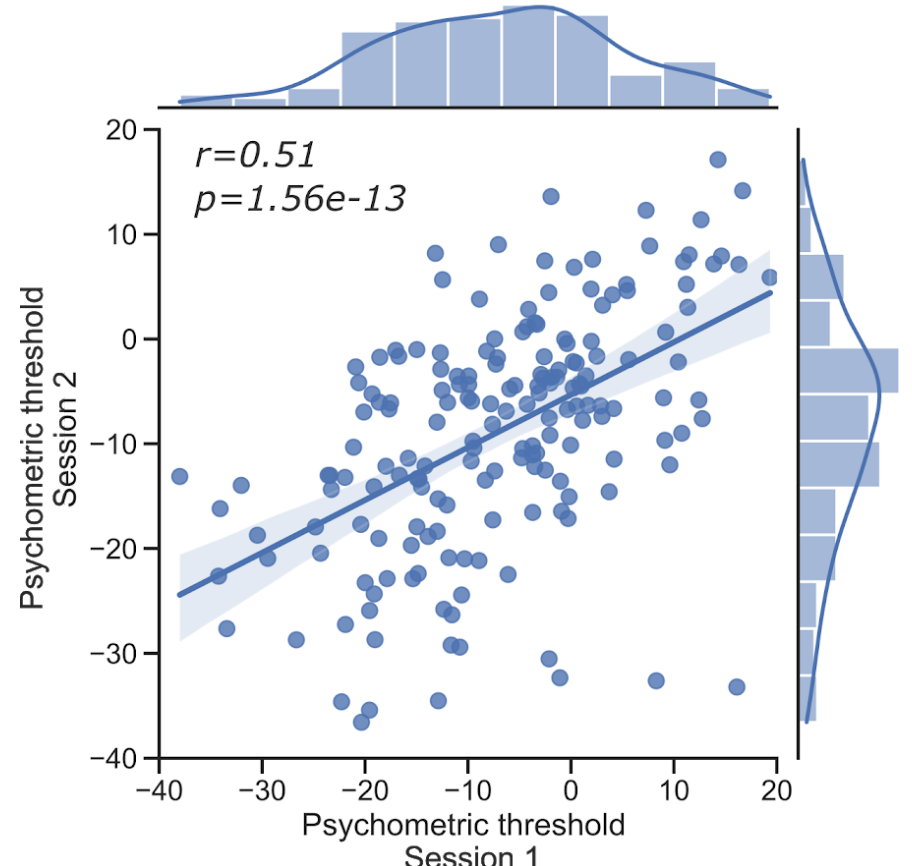

b. Psychometric threshold estimates across modalities and sessions

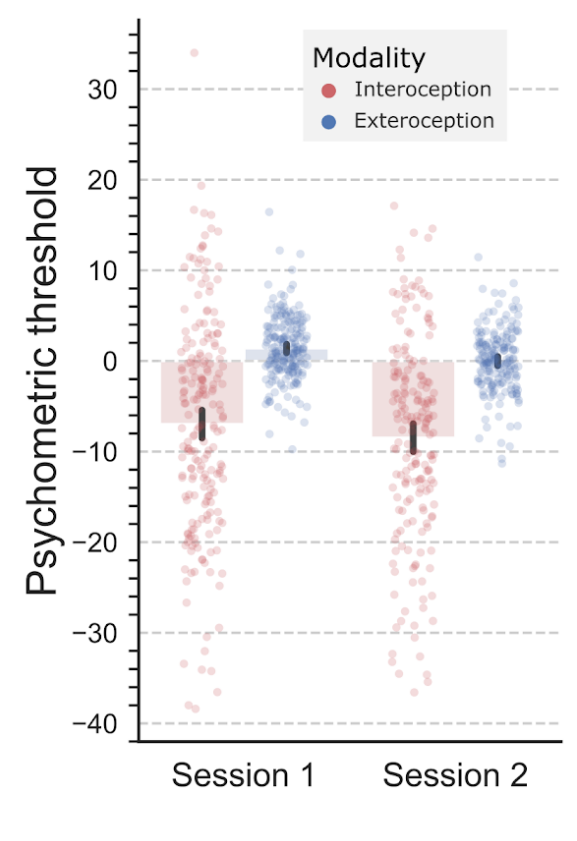

Legend Figure 6: Test-retest reliability of the psychometric threshold. The psychometric threshold estimated using a Bayesian post hoc approach provided correct test-retest reliability. A. Correlation between the interoception threshold estimates in Sessions 1 and 2. Outliers detected by the skipped correlation were removed and the reliability was tested using a Pearson correlation. The $r_{s}$ and $p$ values were calculated using the bootstrapped Spearman coefficient. The regression line was only fitted to non-outlier data points. The shaded area represents the bootstrapped confidence interval (95\%). B. Distribution of threshold Bayesian estimates across sessions and modalities ( $\mathrm{n}=204$ for Session $1 ; \mathrm{n}=190$ for Session 2). 


\section{Discussion}

The measurement of cardiac interoception is a methodological puzzle that has challenged generations of psychologists and psychophysiologists (Ainley et al., 2020; Brener Jasper \& Ring Christopher, 2016; Chen et al., 2021; Zamariola et al., 2018; Zimprich et al., 2020). Here, we suggest that this difficulty arises in part from a reluctance to treat subjective beliefs about the heart rate as a core component of interoception. To remedy this gap, we introduce a new Heart Rate Discrimination (HRD) task, which incorporates a Bayesian psychophysical procedure for measuring the accuracy, precision, and metacognitive sensitivity of cardiac beliefs. In a study of around 200 healthy participants, we observed robust and consistent heart rate underestimation. We also found that interoceptive beliefs and metacognition are more imprecise as compared to a closely matched exteroceptive control condition. Our results indicate that interoceptive beliefs as measured by the HRD are not strongly correlated with other exteroceptive temporal beliefs, but share some variability with indexes of interoception measured by the Heartbeat Counting task. In general, these effects were robustly replicated across two testing sessions, with interoceptive thresholds, in particular, exhibiting good within-participant test-retest reliability. These features make the HRD well-suited for the measurement of interoceptive biomarkers in clinical populations, and for basic research probing the underlying mechanisms underlying cardiac beliefs and their influence on behavior.

Our principal finding is that participants consistently underestimate their resting heart rate by $7 \mathrm{BPM}$ on average, with substantial inter-individual variation around this average $(\Delta$-BPM threshold range $=[-39,30])($ Fig. 2$)$. This finding is consistent with repeated reports that heartbeat counting scores are driven by undercounting (Zamariola et al., 2018) - for discussion, see (Ainley et al., 2020; Corneille et al., 2020; Zimprich et al., 2020). We also find that interoceptive HRD thresholds are moderately correlated with HBC AACC scores, such that fewer counted heartbeats correlate with a lower HRD threshold (see Fig. 5). When comparing interoceptive and exteroceptive thresholds, we further found a modest positive correlation $\left(\mathrm{R}_{\text {Session1 }}=0.15, \mathrm{R}_{\text {Session2 }}=0.26\right)$. These results highlight the unique sources of variance influencing interoceptive beliefs, such that HRD thresholds (and by extension, HBC 
scores) are likely to be driven by a combination of general temporal estimation ability, bottom-up cardio-sensory inputs, and top-down beliefs about the heart rate.

The ability to distinguish these contributions is a unique strength of the HRD. Future clinical investigations will benefit from including both interoceptive and exteroceptive conditions in order to tease apart these different potential causes of apparent interoceptive dysfunction. For example, if a participant group shows general cross-domain effects on both interoceptive and exteroceptive thresholds, this would indicate a general deficit in temporal estimation rather than an alteration of interoceptive beliefs. In contrast, group or condition effects on interoceptive threshold, slope, or sensitivity in the absence of any exteroceptive effects would indicate a specific deficit in monitoring bodily sensations and updating cardiac beliefs. In this way, investigating condition by group interactions on HRD parameters should hopefully improve the specificity of interoception research.

What might underlie this tendency towards underestimation? A critical point is that participants perform our experiment, and most typical cardiac interoception experiments, while seated at a resting heart rate. Recent calls for improved interoceptive biomarkers highlight the possibility that interoceptive processing likely differs depending on whether a participant is in a state of rest versus cardiovascular arousal (Khalsa et al., 2018). In general, cardiac sensations are attenuated at rest, suggesting that participants are more likely to rely on other factors such as prior beliefs when estimating the resting heart rate. Our task quantifies this effect, and raises questions as to what psychological or evolutionary function such a prior may serve. To delineate the physiological and psychological sources of this underestimation bias, a key goal of future experiments will be to manipulate both cardiac sensory afferents (e.g., by pharmacologically or experimentally inducing heart rate acceleration), and prior beliefs about the heart rate (e.g., through probabilistic cues paired with arousing stimuli). Such an experiment can reveal the extent to which these sources of information influence cardiac estimation, and could also provide useful markers of individual differences in interoceptive belief updating.

Another important finding is that interoceptive precision, as measured by the slope of the psychometric function, was substantially lower than exteroceptive precision (Fig 2 and Supp Fig. 1). This is an interesting finding in light of recent theoretical and computational models which hypothesize that interoceptive sensory signals in the brain may generally be more imprecise than their exteroceptive counterparts (Ainley et al., 2016; Allen et al., 2019; 
Allen \& Tsakiris, 2018). It should be noted however that "precision" as measured by the HRD indicates the uncertainty of the psychological decision process, and should not yet be treated as a direct read-out or measurement of the computational process by which prediction error signals are "precision-weighted", which is thought to depend on neurobiological gain control (Bastos et al., 2012; Feldman \& Friston, 2010). While previous investigations in the exteroceptive domain demonstrated a link between behavioral variability of this sort and neurocomputational precision (Eldar et al., 2013; Hénaff et al., 2020; van Bergen et al., 2015; Warren et al., 2016), in advance of direct evidence in the interoceptive domain this link should be interpreted with caution. Nevertheless, a unique benefit of our approach is that future studies could combine the HRD with computational modelling and direct neuronal recordings to conclusively establish the potential link between these parameters.

We also observed substantial test-retest effects on interoceptive precision, such that uncertainty was robustly reduced across sessions. This finding, paired with the observation of poor within-subject reliability for this measure, suggests that interoceptive precision may indicate a more state-based process that is highly amenable to learning, rather than a stable trait. However, caution should also be exercised in interpreting these repeated measures effects as they could arise simply from changes made to the task between the two testing sessions, and/or due to observed difficulties in estimating the slope parameter at Session 1

(Supp. Fig. 2). The accurate estimation of the slope of a psychometric function is typically more difficult than the threshold, requiring a larger number of above and below-threshold trials (Kontsevich \& Tyler, 1999).

In general, we advise users wishing to estimate interoceptive precision to run the maximum number of trials they can afford and to apply fully hierarchical modelling to estimate group-level psychometric parameters, as this approach can improve the fit of both threshold and slope. This option will be implemented in future releases of the python cardioception package. A useful goal for future research then is to tease apart the various test-retest effects observed here (e.g., on $d^{\prime}$, confidence, and slope), through randomized control trials of repeated HRD measurements, perhaps in combination with accuracy feedback manipulations. The introduction of feedback will likely improve the participants' HRD performance, and could offer a low-impact method for clinical intervention in populations with interoceptive disruption. 
Finally, we observed a robust reduction in metacognitive efficiency for interoceptive versus exteroceptive beliefs. Although individual levels of subjective confidence (i.e., metacognitive bias) were highly correlated between modalities, metacognitive efficiency itself was not. This speaks to ongoing debates about the modularity of metacognition (Rouault et al., 2018), indicating that metacognitive ability in the interoceptive domain is largely unrelated to exteroceptive self-monitoring, in line with previous findings on this topic (Beck et al., 2019; Garfinkel et al., 2016). In light of these results, it is interesting to speculate as to the divergent mechanisms that might underlie metacognition in these two domains.

Numerous computational accounts emphasize that accurate metacognitive self-monitoring is likely to depend on a process by which the precision of the sensory signals underlying the type 1 decision is "read-out" by a higher-order metacognitive module, such that noisy, imprecise signals can be expected to degrade both perceptual performance and metacognitive sensitivity (Fleming et al., 2012; Maniscalco \& Lau, 2016). However, other accounts emphasize that top-down "self-beliefs" may play a crucial role in shaping the interaction between low-level precision and higher-order metacognition (Allen et al., 2020; Fleming \& Daw, 2017). Speculatively, our findings suggest that in the cardiac domain, metacognition may be largely dominated by top-down beliefs, rather than pure sensory read-out. Alternatively, if the reduced interoceptive precision observed here relates primarily to the uncertainty of cardiac sensory afferents, then this effect may be simply a result of the metacognitive system accurately reading-out the low sensory precision. Teasing apart these different hypotheses through targeted causal manipulations of cardiac sensory signals and belief will hopefully shed new light on metacognitive insight into the bodily self.

\section{Strengths of the Heart Rate Discrimination Task}

The HRD has several important methodological and practical strengths that support its utility in both basic and clinical research. First, the psychometric curve is estimated across trials relative to the ground truth heart rate. This allows us to differentiate the bias from the sensitivity of cardiac beliefs, in a way in which previous tasks such as HBC and HBD cannot. For example, it could be expected that the overall shape of the psychometric function may change under cardiovascular arousal, and the magnitude of this change could be an important marker of inter-individual differences in interoceptive reactivity. 
A second feature of the HRD is the inclusion of a well-matched exteroceptive control condition, enabling measurements in the same units $(\Delta$-BPM) in both modalities. This provision of sensible, easy to interpret units enables precise, meaningful comparisons across different studies, improving metric interpretability. The exteroceptive control condition itself has several additional benefits; it facilitates the use of the task in neuroimaging studies aiming to isolate more specific neural correlates of cardioceptive beliefs and allows for the differentiation of clinical symptoms into specific interoceptive deficits and more general temporal estimation deficits.

A third strength is that up to $100 \mathrm{HRD}$ trials can be collected in as little as 25 minutes using standard physiological recording equipment. This is critical for clinical studies where testing time is often limited. A related benefit of our trial design is that the HRD is by design amenable to a variety of quantitative modelling techniques such as hierarchical modelling of psychometric functions, or through computational modelling using reinforcement learning and similar approaches (Mathys et al., 2014; Petzschner et al., 2021). This feature facilitates testing mechanistic hypotheses about how cardioceptive beliefs are formed and updated and could be paired with, for example, the probabilistic manipulation of accuracy feedback to delineate the role of prior beliefs and sensory prediction errors.

In general, we believe the HRD will be particularly useful as a clinical biomarker when comparing how specific populations update their cardiac beliefs under differing contexts - for example, one could test whether participants with anxiety show a tendency towards overestimating the heart rate at rest, or instead exhibit larger shifts in threshold and/or precision when comparing aroused vs. resting state performance.

\section{Limitations}

The HRD offers several improvements with respect to existing cardioceptive measures, including increased face validity, adaptability, and amenability to signal theoretic and other computational approaches to quantifying cardiac beliefs. However, there are a few potential limitations of the task, and the results demonstrated here.

First, the HRD depends upon the online estimation of the heart rate within a five-second interval. While instantaneous measures of heart rate are generally robust, even within this time window there are likely to be within-trial shifts in high-frequency heart rate 
variability (HRV). Effectively this means that there is a theoretical lower bound on the precision with which one can estimate HRD thresholds, below which their interpretation becomes suspect. To control for this effect, we ensured that HRD step sizes (e.g., in terms of the minimum increment on $\Delta$-BPM) are never lower than 1 BPM intervals, and also excluded trials with an extreme standard deviation of within-trial beat to beat intervals.

Another limitation is related to our implementation of the task as a two-interval forced-choice response. On each trial, participants first attended to their cardiac sensations and were then immediately presented with auditory feedback during the choice interval. This is a deliberate design decision, as the 2-IFC structure both ensures that participants have a window of interoception-only focus on each trial and renders the underlying behaviour more amenable to the signal theoretic assumptions of the metacognitive model (Galvin et al., 2003; Lee et al., 2018; Maniscalco \& Lau, 2012b). We see this as an improvement over measures such as the heartbeat discrimination task, where subjects must perform a difficult simultaneous multisensory judgement, and it makes the task more amenable for identifying the neural or physiological correlates of HRD measures in the interoception-only time window. However, as a trade-off, this does induce a slight working-memory component to the task, as participants must form a belief about the heart rate and then hold it in mind while comparing it to the auditory feedback tones. This may be a limitation for studies comparing, for example, clinical populations with known working memory deficits. In this case, a variant of the task could easily be implemented in which the feedback tone is presented simultaneously with the listening interval, similar to recent tasks using a method-of-adjustment (Palmer et al., 2019).

Finally, we do not present the HRD as measuring the objective sensitivity to ascending (i.e., baroreceptor mediated) cardiac sensations specifically. For researchers targeting specifically visceral ascending sensitivity, we would recommend approaches such as the MCS (Brener et al., 1993). Our task instead measures the accuracy, bias, and precision of subjective beliefs in the heart rate, which are likely to combine prior beliefs, contextual factors, and ascending (interoceptive and exteroceptive) sensory information where available. Future studies will pair manipulations of ascending cardiac signals with threshold measurement, to better delineate the degree to which these sensory inputs shape cardiac beliefs. 


\section{Conclusion}

In this study, we reported observations from the experimental use of the Heart Rate

Discrimination task to measure the accuracy, bias, and precision of cardiac beliefs among a group of 218 individuals, in a test-retest design. Our results have documented a robust tendency across participants to underestimate their own heart rate, and have shown that interoceptive beliefs are imprecise as compared to an exteroceptive control condition. We argue that the ability to objectively quantify these beliefs is a powerful tool for both basic and clinical interoception research, such that our task offers substantive improvements in the specificity, reliability, and interpretability over existing measures. As this procedure is supported by psychophysics and Bayesian modelling of metacognition, it also calls for future methodological refinement and hypothesis-driven investigation to delineate the computational and physiological sources of cardiac beliefs. 


\section{Acknowledgements}

NL, NN, CMCC, MB, AS, NK, MN, and MA are supported by a Lundbeckfonden Fellowship (under Grant [R272-2017-4345]), and the AIAS-COFUND II fellowship programme that is supported by the Marie Skłodowska-Curie actions under the European Union's Horizon 2020 (under Grant [754513]), and the Aarhus University Research Foundation. FF is supported by an European Research Council Starting Grant, under the European Union's Horizon 2020 research and innovation programme (Grant agreement No. 948838). The authors further thank Benjamin Vincent for insightful discussions on the psychometric approach implemented here. 


\section{Supplementary material}

\section{Psychometric estimates using the Psi method}

During Session 1, we used a 1-up-1-down procedure together with a Psi staircase to estimate the threshold of the psychometric function. During the first 30 trials of each condition, the intensity value was controlled by a 1-up/1-down staircase (Dixon \& Mood, 1948) and the results were provided to the Psi staircase for initialization. We used this procedure to control for threshold convergence between the two techniques (results non reported here). During Session 2, we used only a Psi staircase procedure (Kontsevich \& Tyler, 1999). The experimental setup was also slightly optimized between Sessions 1 and 2 (see Material and Methods). All these points could impact the efficiency and the parameters estimates of the Psi staircases. To check for possible deviation, we report in Figure 1 of the Supplementary Materials the psychometric parameters estimates for slope and threshold across the two modalities and across the two sessions.
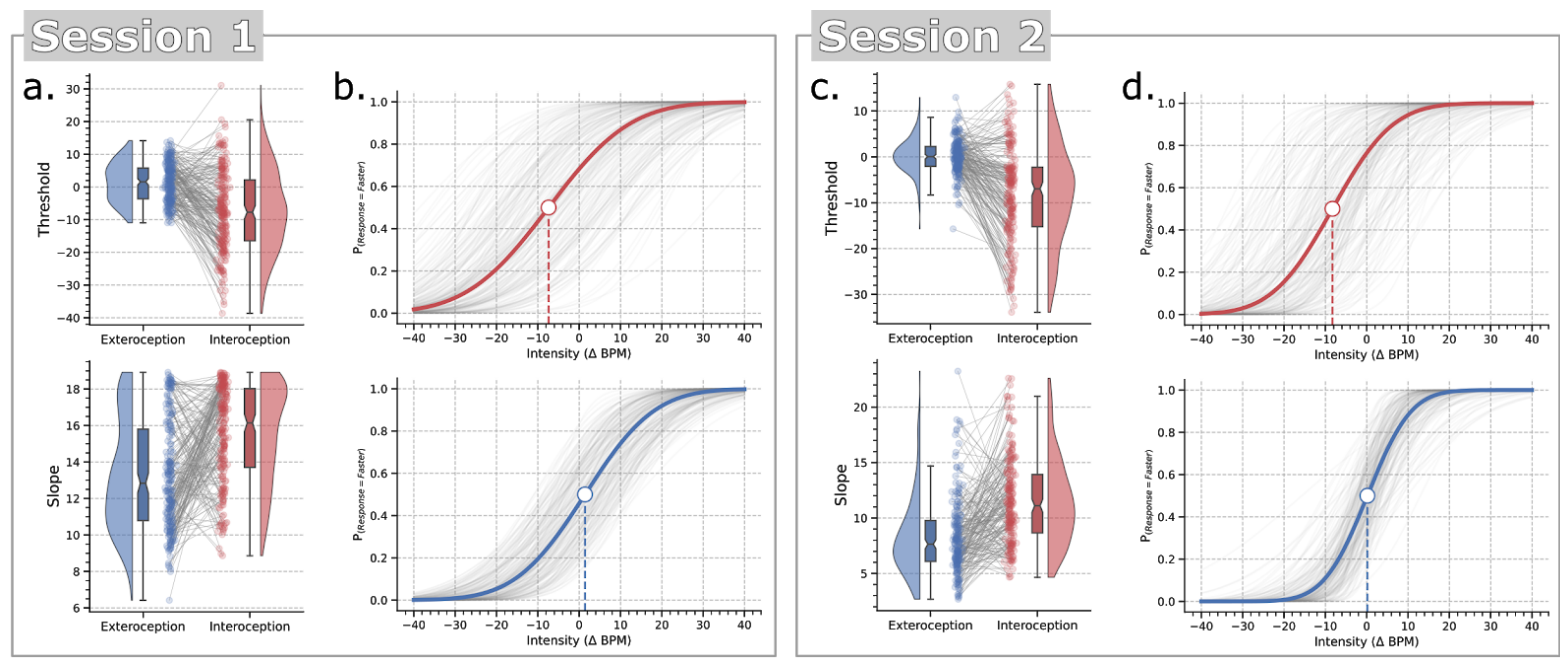

Legend Supplementary Material 1: Psychometric parameters and psychometric functions estimated by the staircase using the Psi method from Sessions 1 and 2. Slope and threshold parameters of the psychometric functions for interoception (red) and exteroception (blue) conditions during Session 1 (n=206) (A.) and Session $2(\mathrm{n}=191)$ (C.). Psychometric functions fitted across interoceptive and exteroceptive conditions for Session 1 (B.) and Session 2 (D.). The grey lines show individual subject fits. The dark blue and red lines show the grand mean psychometric function, depicting average threshold and slope. Both sessions show a strong effect of 
interoception on slope and threshold as compared to the exteroceptive control condition, with a negative bias and reduced precision for interoception.

Here, our results mirrored what we observed using the Bayesian estimates and comparing the two modalities conditions. We observed a bias in the interoceptive threshold as compared to the exteroceptive one in both Session $1\left(\mathrm{t}_{(205)}=-9.89, p<0.001, \mathrm{BF}_{10}=1.20 \mathrm{e}+16\right.$, $d=-0.90)$ and Session $2\left(\mathrm{t}_{(190)}=-11.66, p<0.001, \mathrm{BF}_{10}=8.31 \mathrm{e}+20, d=-1.06\right)$. The slope, reflecting the imprecision of the decision, was also higher during interoception in both Session $1\left(\mathrm{t}_{(205)}=7.86, p<0.001, \mathrm{BF}_{10}=3.06 \mathrm{e}+10, d=0.80\right)$ and Session $2\left(\mathrm{t}_{(190)}=8.92, p<\right.$ $\left.0.001, \mathrm{BF}_{10}=1.50 \mathrm{e}+13, d=0.86\right)$. Here, a higher slope reflects a less precise decision process. These results suggest that the two main psychometric effects (i.e., the threshold bias and slope increase during interoception) are robust and are not specific to one analytical approach in particular.

\section{Correlation between psychometric parameters estimated using the Psi method and a Bayesian post hoc model}

In this paper the psychometric parameters were estimated using a Bayesian model fitted on post-processed response data. This provides, in our opinion, a more robust framework for between session comparisons, and has the advantage to allow for behavioral and physiological data cleaning before model fitting. However, the values of the parameters can also differ between the Psi procedure and the final Bayesian estimates. We report in Figure 2 of the Supplementary Materials the relation between the values estimated by these two methods for both sessions.

When testing covariance using a Pearson correlation, we observed that the threshold estimates were highly consistent across the two estimation methods in both Session 1 (Exteroception: $\mathrm{r}=0.63, \mathrm{CI}_{95 \%}=[0.55,0.71], \mathrm{n}=206$; Interoception: $\mathrm{r}=0.92, \mathrm{CI}_{95 \%}=[0.91$, 0.94], $\mathrm{n}=206$ ) and Session 2 (Exteroception: $\mathrm{r}=0.96, \mathrm{CI}_{95 \%}=[0.95,0.97], \mathrm{n}=154$; Interoception: $\left.\mathrm{r}=0.98, \mathrm{CI}_{95 \%}=[0.98,0.99], \mathrm{n}=147\right)$. These effects are illustrated in

\section{Supplementary Material Fig 2. a-c.}

We observed more variability in the estimation of slope, as reflected by the slightly lower correlation coefficients in Session 1 (Exteroception: $\mathrm{r}=0.69, \mathrm{CI}_{95 \%}=[0.62,0.76], \mathrm{n}=$ 
206; Interoception: $\left.\mathrm{r}=0.80, \mathrm{CI}_{95 \%}=[0.75,0.84], \mathrm{n}=206\right)$ compared to Session 2 (Exteroception: $\mathrm{r}=0.90, \mathrm{CI}_{95 \%}=[0.87,0.93], \mathrm{n}=154$; Interoception: $\mathrm{r}=0.78, \mathrm{CI}_{95 \%}=[0.71$, $0.84], \mathrm{n}=147)$. Notably, a ceiling effect and a systematic shift of the slope estimates was observed on Session 1 (see Supplementary Material Fig 2. b-d). The ceiling effect was corrected in Session 2 by using larger parameter ranges. Here, the Bayesian approach included a larger prior range and was able to infer different slope values when the maximum was reached.

This analysis illustrates the power of a simple post hoc Bayesian modelling approach to improve and correct potential issues in the settings of the Psi staircase. This approach can be further expanded in future works, for example using fully hierarchical (i.e., mixed-effects) Bayesian modelling across participants and groups, improving the estimation of conditional differences in threshold or slope values. This could enhance statistical power by pooling and it further limits the influence of unlikely or outlier responses through group shrinkage effects on the parameter estimates.
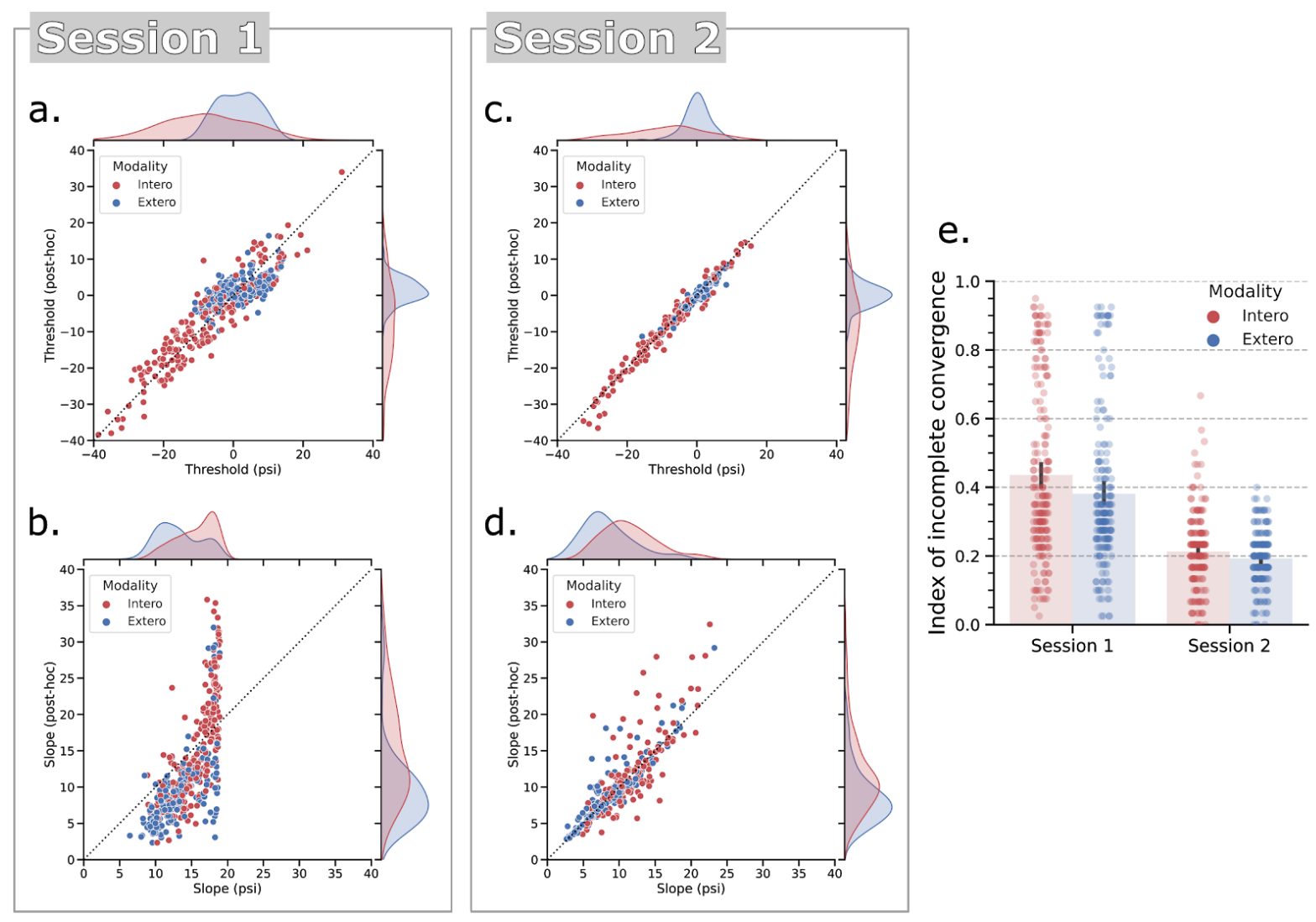

Legend Supplementary Material 2: Comparison between online and post hoc Bayesian estimation of slope and threshold parameters of the psychometric functions. Adaptive Bayesian staircases can be biased if their 
initial parameter settings poorly fit the underlying generative psychometric function, or if a subject makes unrepresentative responses early in the experiment. For example, in this sample we observed that the prior width [0 - 20] on the slope parameter was too low, resulting in a ceiling effect that biased our estimates in a subset of participants. One solution to control these biases is to implement post hoc Bayesian modelling of the observed psychophysical data. We thus re-analyzed the responses for each participant and for each condition separately using a Bayesian model to fit a cumulative normal distribution. A. The thresholds estimates remained stable, although with a reduced variance for the exteroceptive condition. B. The ceiling effect on the slope were normalized by the post hoc modelling, which shifts the posterior mass away from the extremes. The post hoc procedure can thus improve the estimation of the interoceptive and exteroceptive psychophysical parameters.

Another reason for using a Bayesian model was the presence of incomplete convergence of the Psi staircase during the first session. The Psi algorithm (Kontsevich \& Tyler, 1999) is designed to test intensity values that would first increase the precision of the posterior density for threshold. When this confidence around the threshold level is high enough, the staircase starts to improve precision for the slope estimate by testing intensity values around the threshold. This results in a recognizable pattern of higher and lower intensities values alternating regularly around the inferred threshold. Interfering with the Bayesian updating during the first 30 trials of the task, as in Session 1, could result in biased estimation of threshold values. Further, erroneous responses during the first trials may hinder convergence.

Here, we quantified the amount of incomplete Psi staircase convergence through the two sessions. Incomplete convergence is characterized by stimulus intensity values that are consistently higher or lower than the inferred threshold even at the end of the task. To quantify this effect, we calculated an incomplete convergence index using the ratio of high intensity versus equal or low intensity values with respect to the inferred threshold in the last 40 trials. This ratio was then converted using the following formula:

$$
\text { IncompleteConvergence }=\mid \text { ratio }-0.5 \mid * 2
$$

This formula returns a real number between 0 and 1.0 indicates that the intensity values were equally distributed around the inferred threshold in the last 40 trials. Instead, 1 indicates divergence between the tested intensity values and the inferred threshold. The incomplete convergence indexes for Interoception and Exteroception through Session 1 and 2 are reported in Supplementary Material Fig 2. e). These results revealed a high proportion of incomplete convergence in the first session, in both interoception and exteroception 
conditions. For example, setting an arbitrary threshold for quality assessment at 0.5 revealed that 63 and 41 participants had poor convergence for interoception and exteroception, respectively. These numbers dropped radically in Session 2 (see Material and Method) and corresponded to only 3 and 0 staircases for interoception and exteroception, respectively. The improved convergence in Session 2 is likely due to the introduction of different design choices, aimed at solving the convergence issues observed in Session 1.

\section{Psychometric results (Session 2)}

We reproduced the approach used in the first session and compared threshold and slope values between the interoception and the exteroception conditions. This revealed that during interoception participants had significantly lower psychometric thresholds $\left(\operatorname{mean}_{\text {Intero }}=-8.50\right.$, $\mathrm{CI}_{95 \%}[-10.06,-6.92]$, mean $n_{\text {Extero }}=0.01, \mathrm{CI}_{95 \%}[-0.47,0.52], \mathrm{t}_{(190)}=-11.15, \mathrm{p}<0.001, \mathrm{BF}_{10}=$ $2.85 \mathrm{e}+19, \mathrm{~d}=-1.03)$ and higher psychometric slopes $\left(\operatorname{mean}_{\text {Intero }}=11.96, \mathrm{CI}_{95 \%}[11.22,12.74]\right.$, mean $\left._{\text {Extero }}=8.69, \mathrm{CI}_{95 \%}[8.14,9.28], \mathrm{t}_{(190)}=-7.29, \mathrm{p}<0.001, \mathrm{BF}_{10}=9.12 \mathrm{e}+08, \mathrm{~d}=0.67\right)$. See Figure 2 and Supplementary Figure 1 for illustration of these effects. Similarly to the results in the first session, the negative bias of the threshold parameters suggests that participants underestimated their heart rate on average. The greater slope on the other side, indicates a less precise decision process. 
bioRxiv preprint doi: https://doi.org/10.1101/2021.02.18.431871; this version posted February 18, 2021. The copyright holder for this preprint (which was not certified by peer review) is the author/funder, who has granted bioRxiv a license to display the preprint in perpetuity. It is made available under aCC-BY-NC-ND 4.0 International license.

a. Psychometric parameters
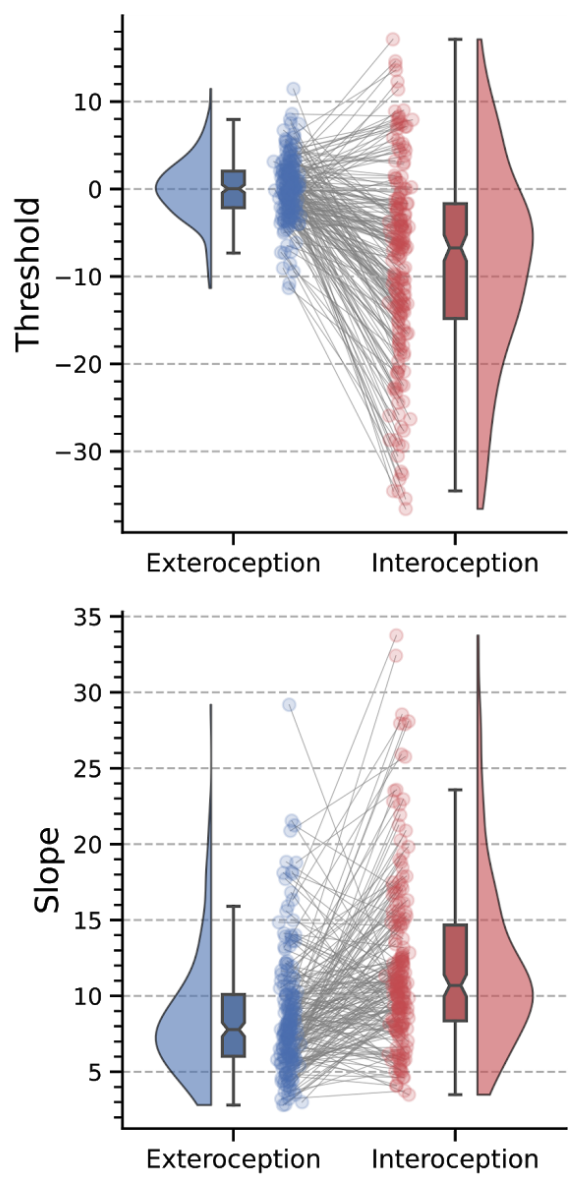

b. Psychometric functions
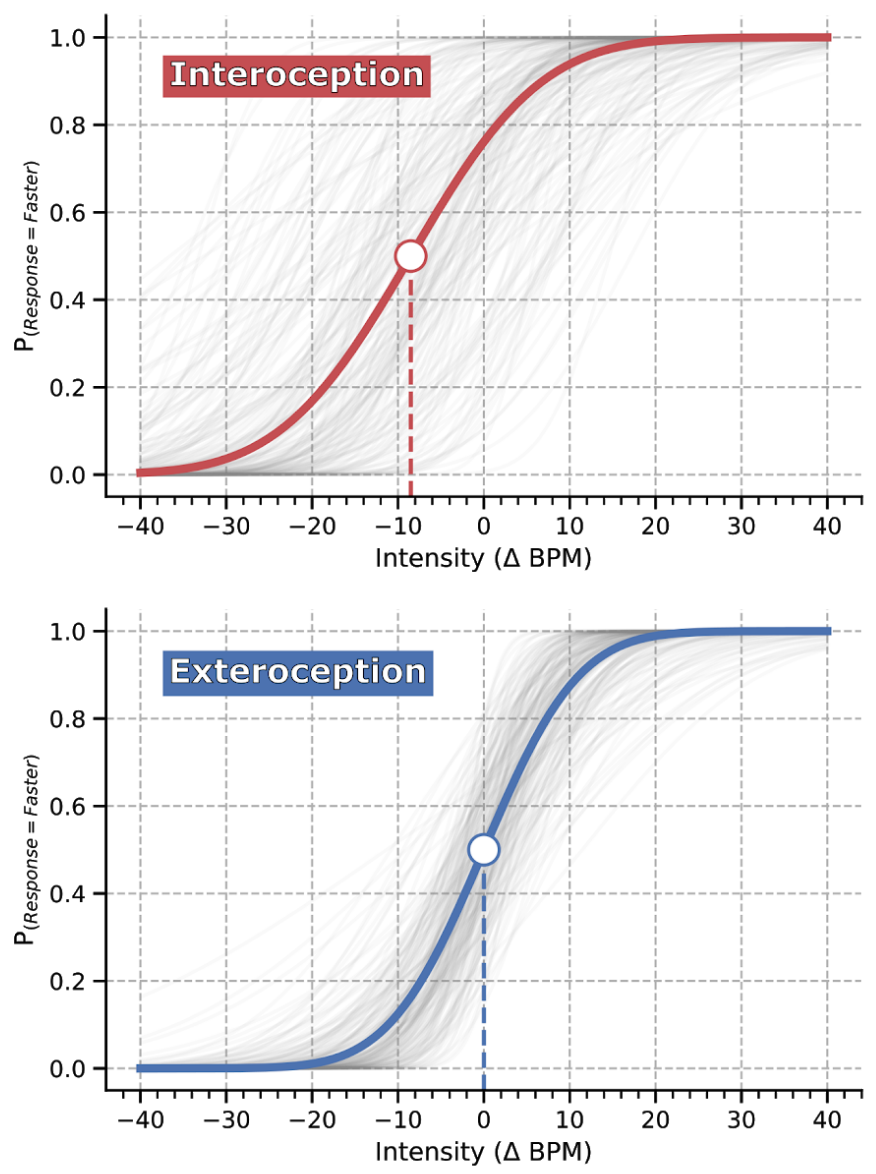

Legend Supplementary Material 3: Psychometric parameter estimates and fitted interoception and exteroception psychometric functions (Session 2). A. Repeated measures raincloud plots visualizing threshold and slope parameters of the psychometric functions across the two modalities (interoception and exteroception). Data points for every individual are connected by a grey line to highlight the repeated measure effect. B. The grey lines show individual subject fits. The dark red and blue lines show the grand mean psychometric function, depicting averaged threshold and slope. Grand mean thresholds are marked by the large point, where the psychometric function crosses 0.5 on the ordinate axis. We observed a strong effect of interoception on both slope and threshold parameters as compared to the exteroceptive control condition.

\section{Metacognition results (Session 2)}

The $d$, which reflects discimination sensitivity, was lower in the interoception condition $\left(\operatorname{mean}_{\text {Intero }}=1.88, \mathrm{CI}_{95 \%}=[1.78,1.96]\right.$, mean $_{\text {Extero }}=2.25, \mathrm{CI}_{95 \%}=[2.21,2.3], t_{(189)}=-8.10, p<$ $\left.0.001, \mathrm{BF}_{10}=9.67 \mathrm{e}+10, d=-0.77\right)$. Further, as in the first session, we found that metacognitive sensitivity was significantly lower during interoception. The interoceptive M-ratio estimates were lower $\left(\operatorname{mean}_{\text {Intero }}=0.83, \mathrm{CI}_{95 \%}=[0.8,0.87]\right)$ than the exteroceptive 
ones $\left(\operatorname{mean}_{\text {Extero }}=0.96, \mathrm{CI}_{95 \%}=[0.92,1.01]\right)$. The posterior distribution of the repeated measure effect was also lower (mean $=-0.17 \mathrm{HDI}_{94 \%}=[-0.28,-0.05]$.

\section{a. Confidence ratings for correct and incorrect responses}
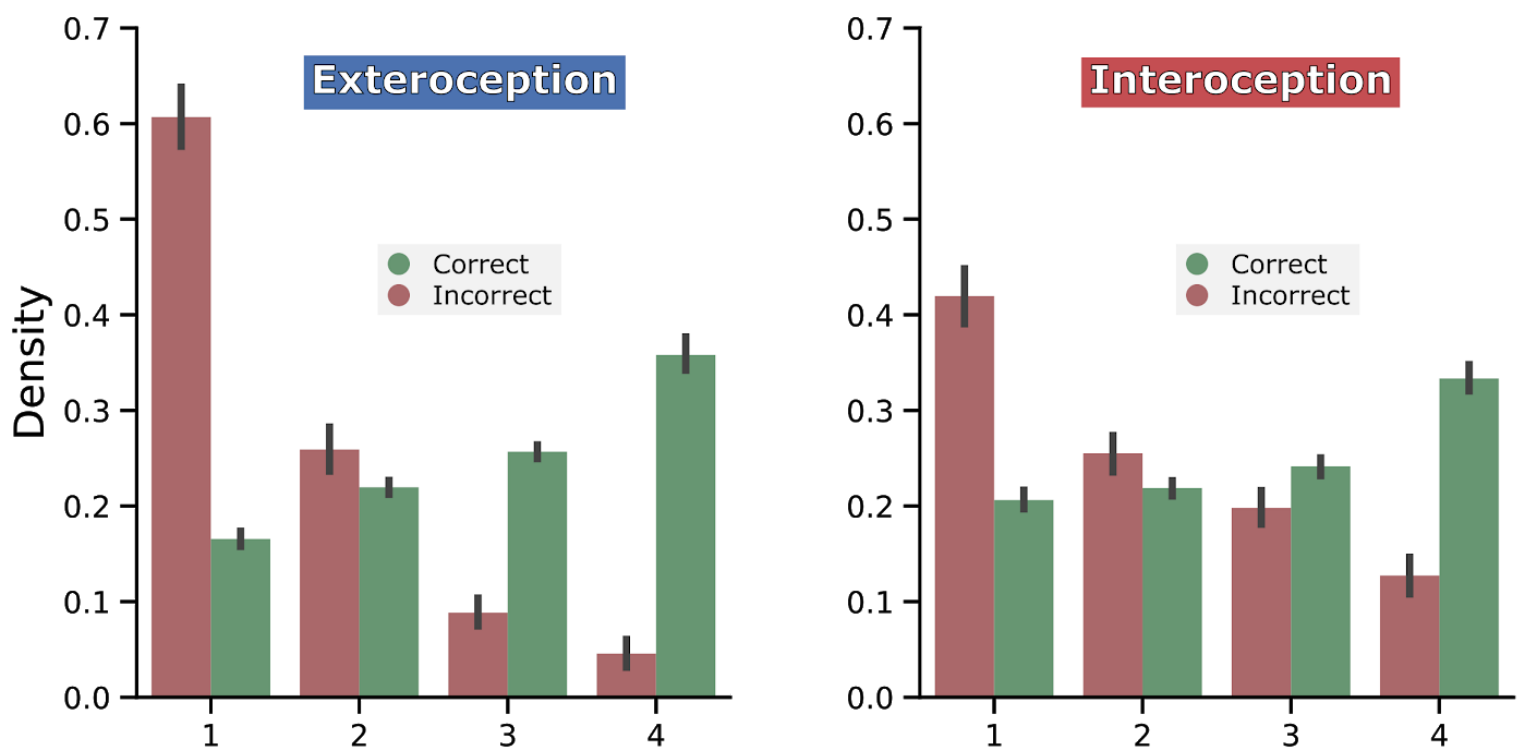

\section{b. Metacognitive efficiency (m-ratio)}

c. Effect of interoception on metacognitive efficiency
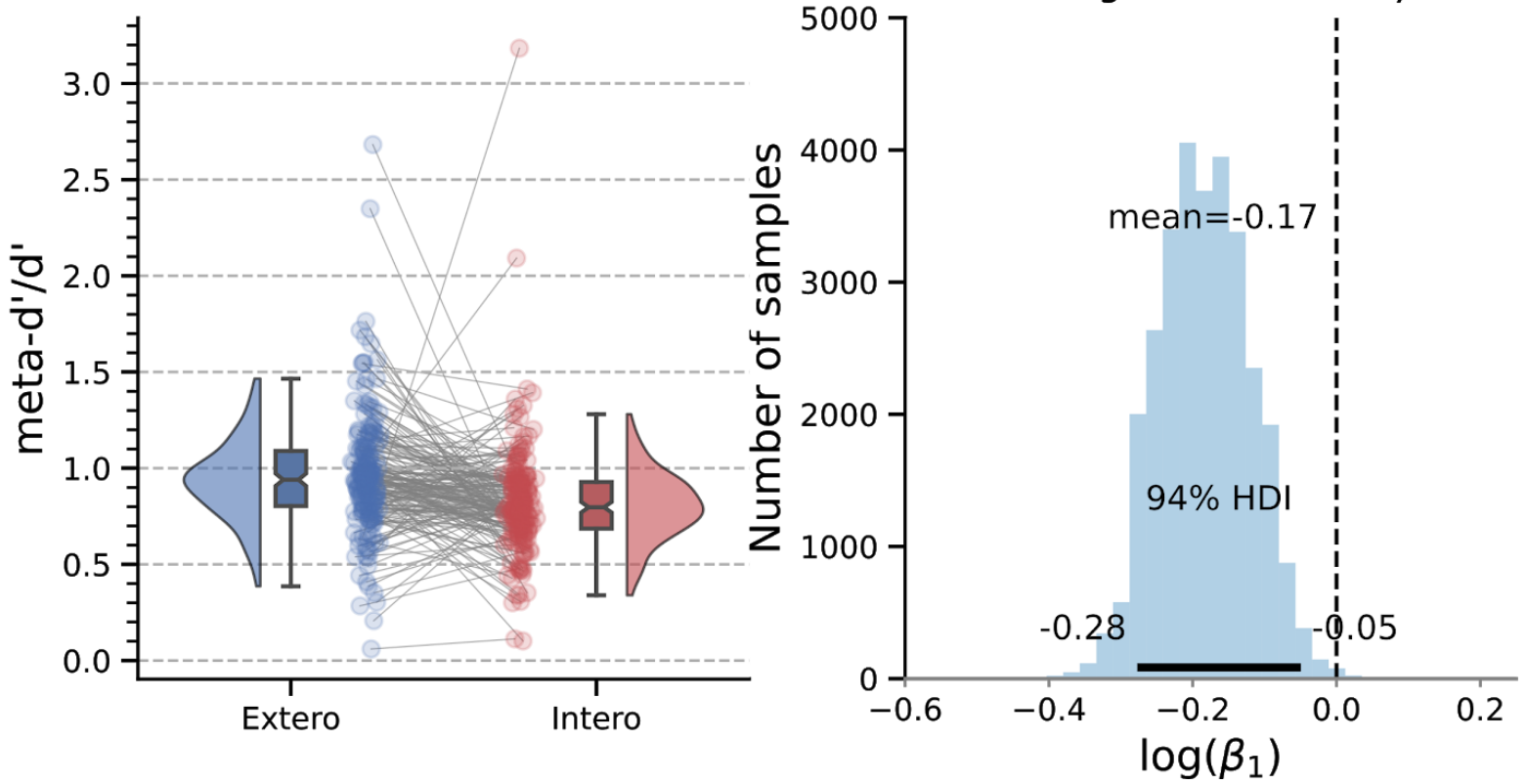

Legend Supplementary Material 3: Visualization of metacognitive performance for interoceptive and

exteroceptive conditions (Session 2). A. Histogram showing the distribution of binned confidence ratings for correct vs. error trials. Participants were significantly less confident overall in the interoceptive condition and showed reduced calibration as indicated by the flattening of the confidence distributions. To quantify this effect, we estimated "metacognitive efficiency", a signal theoretic model of introspective accuracy which controls for differences in type 1 (discrimination) performance. Here, an M-ratio of 1 indicates optimal metacognition according to an ideal observer model, whereas values lower than this indicate inefficient use of the available 
bioRxiv preprint doi: https://doi.org/10.1101/2021.02.18.431871; this version posted February 18, 2021. The copyright holder for this preprint (which was not certified by peer review) is the author/funder, who has granted bioRxiv a license to display the preprint in perpetuity. It is made available under aCC-BY-NC-ND 4.0 International license.

perceptual signal. B. This model demonstrated that metacognitive efficiency was substantially decreased for interoceptive relative to exteroceptive judgements. C. Histogram of posterior samples from the beta value coding the effect of interoception.

\section{Cross-modal correlation (Session 2)}

We observed more robust and consistent correlations between task parameters within each modality (interoception or exteroception), but few significant correlations between task modalities, indicating a high degree of independence between performance on the two task conditions. Across modalities, response times during the decision process (type 1 measure) were correlated between the interoception and the exteroception conditions $\left(\mathrm{r}_{\mathrm{s}}=0.66, \mathrm{CI}_{95 \%}=\right.$ $\left.[0.58,0.74], p<0.001, \mathrm{n}=190, \mathrm{n}_{\text {outliers }}=5\right)$, as well as between confidence ratings $\left(\mathrm{r}_{\mathrm{s}}=0.54\right.$, $\left.\mathrm{CI}_{95 \%}=[0.44,0.64], \mathrm{p}<0.001, \mathrm{n}=190, \mathrm{n}_{\text {outliers }}=3\right)$.

\section{Cross-modal correlations matrix}

FDR corrected $(p<0.01)$

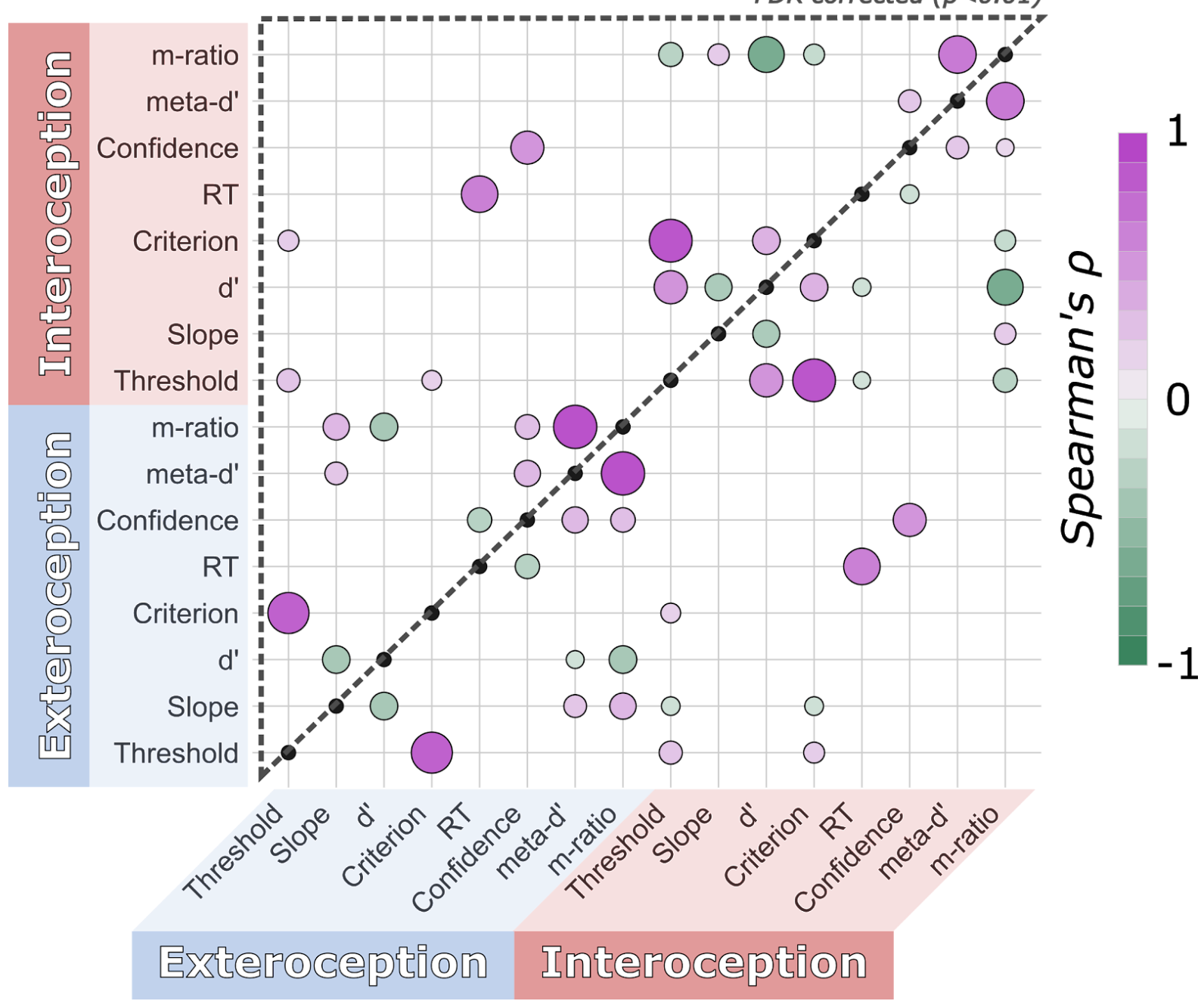


bioRxiv preprint doi: https://doi.org/10.1101/2021.02.18.431871; this version posted February 18,2021 . The copyright holder for this

preprint (which was not certified by peer review) is the author/funder, who has granted bioRxiv a license to display the preprint in perpetuity. It is made available under aCC-BY-NC-ND 4.0 International license.

\section{Legend Supplementary Material 4: Cross-modal correlation heatmap of task parameters for} interoception and exteroception conditions (Session 2). We replicated several of the observations reported in Session 1. Behavioural results were correlated within modalities but with limited dependence across modalities. The only exceptions, already observed in Session 1, were confidence ratings and response times (RT). The figure depicts significant skipped Spearman correlations. The upper triangle shows results surviving FDR correction $\left(\mathrm{p}_{\mathrm{FDR}}<0.01\right)$. Colour and size of individual points indicate the sign and strength of the estimated correlation coefficients. 


\section{References}

Ainley, V., Apps, M. A. J., Fotopoulou, A., \& Tsakiris, M. (2016). 'Bodily precision': A predictive coding account of individual differences in interoceptive accuracy. Philosophical Transactions of the Royal Society B: Biological Sciences, 371(1708), 20160003. https://doi.org/10.1098/rstb.2016.0003

Ainley, V., Tsakiris, M., Pollatos, O., Schulz, A., \& Herbert, B. M. (2020). Comment on "Zamariola et al. (2018), Interoceptive Accuracy Scores are Problematic: Evidence from Simple Bivariate Correlations"- The empirical data base, the conceptual reasoning and the analysis behind this statement are misconceived and do not support the authors' conclusions. Biological Psychology, 152, 107870. https://doi.org/10.1016/j.biopsycho.2020.107870

Allen, M. (2020). Unravelling the Neurobiology of Interoceptive Inference. Trends in Cognitive Sciences, 24(4), 265-266. https://doi.org/10.1016/j.tics.2020.02.002

Allen, M., \& Friston, K. J. (2018). From cognitivism to autopoiesis: Towards a computational framework for the embodied mind. Synthese, 195(6), 2459-2482. https://doi.org/10.1007/s11229-016-1288-5

Allen, M., Legrand, N., Costa Correa, C. M., \& Fardo, F. (2020). Thinking through prior bodies: Autonomic uncertainty and interoceptive self-inference. Behavioral and Brain Sciences, 43, e91. https://doi.org/10.1017/S0140525X19002899

Allen, M., Levy, A., Parr, T., \& Friston, K. J. (2019). In the Body's Eye: The Computational Anatomy of Interoceptive Inference [Preprint]. Neuroscience. https://doi.org/10.1101/603928

Allen, M., Poggiali, D., Whitaker, K., Marshall, T. R., van Langen, J., \& Kievit, R. A. (2021). Raincloud plots: A multi-platform tool for robust data visualization. Wellcome Open Research, 4, 63. https://doi.org/10.12688/wellcomeopenres.15191.2

Allen, M., \& Tsakiris, M. (2018). The body as first prior: Interoceptive predictive processing and the primacy of self-models (Vol. 1). Oxford University Press. https://doi.org/10.1093/oso/9780198811930.003.0002

Barrett, L. F., \& Simmons, W. K. (2015). Interoceptive predictions in the brain. Nature Reviews Neuroscience, 16(7), 419-429. https://doi.org/10.1038/nrn3950

Bastos, A. M., Usrey, W. M., Adams, R. A., Mangun, G. R., Fries, P., \& Friston, K. J. (2012). Canonical Microcircuits for Predictive Coding. Neuron, 76(4), 695-711. https://doi.org/10.1016/j.neuron.2012.10.038

Beck, B., Peña-Vivas, V., Fleming, S., \& Haggard, P. (2019). Metacognition across sensory modalities: Vision, warmth, and nociceptive pain. Cognition, 186, 32-41. https://doi.org/10.1016/j.cognition.2019.01.018

Brener, J., Liu, X., \& Ring, C. (1993). A method of constant stimuli for examining heartbeat detection: Comparison with the Brener-Kluvitse and Whitehead methods. Psychophysiology, 30(6), 657-665. https://doi.org/10.1111/j.1469-8986.1993.tb02091.x

Brener, J., \& Ring, C. (2016). Towards a psychophysics of interoceptive processes: The measurement of heartbeat detection. Philosophical Transactions of the Royal Society B: Biological Sciences, 371(1708), 20160015. https://doi.org/10.1098/rstb.2016.0015

Brener Jasper \& Ring Christopher. (2016). Towards a psychophysics of interoceptive processes: The measurement of heartbeat detection. Philosophical Transactions of the Royal Society B: Biological Sciences, 371(1708), 20160015. https://doi.org/10.1098/rstb.2016.0015

Chen, W. G., Schloesser, D., Arensdorf, A. M., Simmons, J. M., Cui, C., Valentino, R., Gnadt, J. W., Nielsen, L., Hillaire-Clarke, C. St., Spruance, V., Horowitz, T. S., Vallejo, Y. F., \& Langevin, H. M. (2021). The Emerging Science of Interoception: Sensing, Integrating, Interpreting, and Regulating Signals within the Self. Trends in Neurosciences, 44(1), 3-16. https://doi.org/10.1016/j.tins.2020.10.007

Clemens, W. J. (1979). Assessment, Learning, and Retention of Heart Beat Discrimination. Psychophysiology, 16(4), 333-338. https://doi.org/10.1111/j.1469-8986.1979.tb01472.x

Corneille, O., Desmedt, O., Zamariola, G., Luminet, O., \& Maurage, P. (2020). A heartfelt response to Zimprich et al. (2020), and Ainley et al. (2020)'s commentaries: Acknowledging issues with the HCT would benefit interoception research. Biological Psychology, 152, 107869. https://doi.org/10.1016/j.biopsycho.2020.107869

Cornsweet, T. N. (1962). The Staircase-Method in Psychophysics. The American Journal of Psychology, 75(3), 485-491. JSTOR. https://doi.org/10.2307/1419876

Craig, A. D. (2002). How do you feel? Interoception: the sense of the physiological condition of the body. Nature Reviews Neuroscience, 3(8), 655-666. https://doi.org/10.1038/nrn894

Critchley, H. D., \& Garfinkel, S. N. (2017). Interoception and emotion. Current Opinion in Psychology, 17, 7-14. https://doi.org/10.1016/j.copsyc.2017.04.020 
bioRxiv preprint doi: https://doi.org/10.1101/2021.02.18.431871; this version posted February $18,2021$. The copyright holder for this

preprint (which was not certified by peer review) is the author/funder, who has granted bioRxiv a license to display the preprint in perpetuity. It is made available under aCC-BY-NC-ND 4.0 International license.

Dale, A., \& Anderson, D. (1978). Information Variables in Voluntary Control and Classical Conditioning of Heart Rate: Field Dependence and Heart-Rate Perception. Perceptual and Motor Skills, 47(1), $79-85$. https://doi.org/10.2466/pms.1978.47.1.79

Desmedt, O., Corneille, O., Luminet, O., Murphy, J., Bird, G., \& Maurage, P. (2020). Contribution of Time Estimation and Knowledge to Heartbeat Counting Task Performance under Original and Adapted Instructions. Biological Psychology, 154, 107904. https://doi.org/10.1016/j.biopsycho.2020.107904

Desmedt, O., Houte, M. V. D., Walentynowicz, M., Dekeyser, S., Luminet, O., \& Corneille, O. (2020). A Systematic Review and Meta-analysis on the Association Between Heartbeat Counting Task Performance and Mental Disorders and Their Risk Factors Among Adults. OSF Preprints. https://doi.org/10.31219/osf.io/h3by9

Desmedt, O., Luminet, O., \& Corneille, O. (2018). The heartbeat counting task largely involves non-interoceptive processes: Evidence from both the original and an adapted counting task. Biological Psychology, 138, 185-188. https://doi.org/10.1016/j.biopsycho.2018.09.004

Desmedt, O., Luminet, O., Maurage, P., \& Corneille, O. (2020). What If the Heartbeat Counting Task Required No Measure of Cardiac Activity? PsyArXiv. https://doi.org/10.31234/osf.io/yj5s2

Dixon, W. J., \& Mood, A. M. (1948). A Method for Obtaining and Analyzing Sensitivity Data. Journal of the American Statistical Association, 43(241), 109-126. https://doi.org/10.1080/01621459.1948.10483254

Eggart, M., Lange, A., Binser, M., Queri, S., \& Müller-Oerlinghausen, B. (2019). Major Depressive Disorder Is Associated with Impaired Interoceptive Accuracy: A Systematic Review. Brain Sciences, 9(6), 131. https://doi.org/10.3390/brainsci9060131

Eldar, E., Cohen, J. D., \& Niv, Y. (2013). The effects of neural gain on attention and learning. Nature Neuroscience, 16(8), 1146-1153. https://doi.org/10.1038/nn.3428

Feldman, H., \& Friston, K. J. (2010). Attention, Uncertainty, and Free-Energy. Frontiers in Human Neuroscience, 4. https://doi.org/10.3389/fnhum.2010.00215

Fleming, S. M. (2017). HMeta-d: Hierarchical Bayesian estimation of metacognitive efficiency from confidence ratings. Neuroscience of Consciousness, 2017(1). https://doi.org/10.1093/nc/nix007

Fleming, S. M., \& Daw, N. D. (2017). Self-evaluation of decision-making: A general Bayesian framework for metacognitive computation. Psychological Review, 124(1), 91-114. https://doi.org/10.1037/rev0000045

Fleming, S. M., Dolan, R. J., \& Frith, C. D. (2012). Metacognition: Computation, biology and function. Philosophical Transactions of the Royal Society B: Biological Sciences, 367(1594), 1280-1286. https://doi.org/10.1098/rstb.2012.0021

Fleming, S. M., \& Lau, H. C. (2014). How to measure metacognition. Frontiers in Human Neuroscience, 8. https://doi.org/10.3389/fnhum.2014.00443

Flynn, D. M., \& Clemens, W. J. (1988). On the Validity of Heartbeat Tracking Tasks. Psychophysiology, 25(1), 92-96. https://doi.org/10.1111/j.1469-8986.1988.tb00965.x

Forkmann, T., Volz-Sidiropoulou, E., Helbing, T., Drüke, B., Mainz, V., Rath, D., Gauggel, S., \& Teismann, T. (2019). Sense it and use it: Interoceptive accuracy and sensibility in suicide ideators. BMC Psychiatry, 19(1), 334. https://doi.org/10.1186/s12888-019-2322-1

Galvin, S. J., Podd, J. V., Drga, V., \& Whitmore, J. (2003). Type 2 tasks in the theory of signal detectability: Discrimination between correct and incorrect decisions. Psychonomic Bulletin \& Review, 10(4), 843-876. https://doi.org/10.3758/BF03196546

García-Pérez, M. A. (2014). Adaptive psychophysical methods for nonmonotonic psychometric functions. Attention, Perception, \& Psychophysics, 76(2), 621-641. https://doi.org/10.3758/s13414-013-0574-2

Garfinkel, S. N., Manassei, M. F., Hamilton-Fletcher, G., In den Bosch, Y., Critchley, H. D., \& Engels, M. (2016). Interoceptive dimensions across cardiac and respiratory axes. Philosophical Transactions of the Royal Society B: Biological Sciences, 371(1708), 20160014. https://doi.org/10.1098/rstb.2016.0014

Garfinkel, S. N., Seth, A. K., Barrett, A. B., Suzuki, K., \& Critchley, H. D. (2015). Knowing your own heart: Distinguishing interoceptive accuracy from interoceptive awareness. Biological Psychology, 104, 65-74. https://doi.org/10.1016/j.biopsycho.2014.11.004

Hart, N., McGowan, J., Minati, L., \& Critchley, H. D. (2013). Emotional Regulation and Bodily Sensation: Interoceptive Awareness Is Intact in Borderline Personality Disorder. Journal of Personality Disorders, 27(4), 506-518. https://doi.org/10.1521/pedi_2012_26_049

Hénaff, O. J., Boundy-Singer, Z. M., Meding, K., Ziemba, C. M., \& Goris, R. L. T. (2020). Representation of visual uncertainty through neural gain variability. Nature Communications, 11(1), 2513. https://doi.org/10.1038/s41467-020-15533-0

Hoffman, M. D., \& Gelman, A. (2011). The No-U-Turn Sampler: Adaptively Setting Path Lengths in Hamiltonian Monte Carlo. ArXiv:1111.4246 [Cs, Stat]. http://arxiv.org/abs/1111.4246

Hunter, J. D. (2007). Matplotlib: A 2D Graphics Environment. Computing in Science \& Engineering, 9(3), 
90-95. https://doi.org/10.1109/MCSE.2007.55

Khalsa, S. S., Adolphs, R., Cameron, O. G., Critchley, H. D., Davenport, P. W., Feinstein, J. S., Feusner, J. D., Garfinkel, S. N., Lane, R. D., Mehling, W. E., Meuret, A. E., Nemeroff, C. B., Oppenheimer, S., Petzschner, F. H., Pollatos, O., Rhudy, J. L., Schramm, L. P., Simmons, W. K., Stein, M. B., ... Zucker, N. (2018). Interoception and Mental Health: A Roadmap. Biological Psychiatry: Cognitive Neuroscience and Neuroimaging, 3(6), 501-513. https://doi.org/10.1016/j.bpsc.2017.12.004

Khalsa, S. S., \& Lapidus, R. C. (2016). Can Interoception Improve the Pragmatic Search for Biomarkers in Psychiatry? Frontiers in Psychiatry, 7. https://doi.org/10.3389/fpsyt.2016.00121

Kingdom, F. A. A., \& Prins, N. (2016). Psychophysics: A practical introduction (Second edition). Elsevier/Academic Press.

Kontsevich, L. L., \& Tyler, C. W. (1999). Bayesian adaptive estimation of psychometric slope and threshold. Vision Research, 39(16), 2729-2737. https://doi.org/10.1016/S0042-6989(98)00285-5

Kumar, R., Carroll, C., Hartikainen, A., \& Martin, O. (2019). ArviZ a unified library for exploratory analysis of Bayesian models in Python. Journal of Open Source Software, 4(33), 1143. https://doi.org/10.21105/joss.01143

Lee, A. L. F., Ruby, E., Giles, N., \& Lau, H. (2018). Cross-Domain Association in Metacognitive Efficiency Depends on First-Order Task Types. Frontiers in Psychology, 9. https://doi.org/10.3389/fpsyg.2018.02464

Legrand, N., \& Allen, M. (2020). Systole: V 0.1.2-September 2020 (0.1.2) [Computer software]. Zenodo. https://doi.org/10.5281/ZENODO.3607912

Leys, C., Ley, C., Klein, O., Bernard, P., \& Licata, L. (2013). Detecting outliers: Do not use standard deviation around the mean use absolute deviation around the median. Journal of Experimental Social Psychology, 49(4), 764-766. https://doi.org/10.1016/j.jesp.2013.03.013

Lipponen, J. A., \& Tarvainen, M. P. (2019). A robust algorithm for heart rate variability time series artefact correction using novel beat classification. Journal of Medical Engineering \& Technology, 43(3), 173-181. https://doi.org/10.1080/03091902.2019.1640306

Maniscalco, B., \& Lau, H. (2012a). A signal detection theoretic approach for estimating metacognitive sensitivity from confidence ratings. Consciousness and Cognition, 21(1), 422-430. https://doi.org/10.1016/j.concog.2011.09.021

Maniscalco, B., \& Lau, H. (2012b). A signal detection theoretic approach for estimating metacognitive sensitivity from confidence ratings. Consciousness and Cognition, 21(1), 422-430. https://doi.org/10.1016/j.concog.2011.09.021

Maniscalco, B., \& Lau, H. (2016). The signal processing architecture underlying subjective reports of sensory awareness. Neuroscience of Consciousness, 2016(1). https://doi.org/10.1093/nc/niw002

Mathys, C. D., Lomakina, E. I., Daunizeau, J., Iglesias, S., Brodersen, K. H., Friston, K. J., \& Stephan, K. E. (2014). Uncertainty in perception and the Hierarchical Gaussian Filter. Frontiers in Human Neuroscience, 8. https://doi.org/10.3389/fnhum.2014.00825

Mazancieux, A., Fleming, S. M., Souchay, C., \& Moulin, C. J. A. (2020). Is there a G factor for metacognition? Correlations in retrospective metacognitive sensitivity across tasks. Journal of Experimental Psychology: General. https://doi.org/10.1037/xge0000746

Murphy, J., Brewer, R., Coll, M.-P., Plans, D., Hall, M., Shiu, S. S., Catmur, C., \& Bird, G. (2019). I feel it in my finger: Measurement device affects cardiac interoceptive accuracy. Biological Psychology, 148, 107765. https://doi.org/10.1016/j.biopsycho.2019.107765

Murphy, J., Millgate, E., Geary, H., Ichijo, E., Coll, M.-P., Brewer, R., Catmur, C., \& Bird, G. (2018). Knowledge of resting heart rate mediates the relationship between intelligence and the heartbeat counting task. Biological Psychology, 133, 1-3. https://doi.org/10.1016/j.biopsycho.2018.01.012

Palmer, C., Ainley, V., \& Tsakiris, M. (2019). Fine Tuning Your Heart: A novel method for measuring interoceptive accuracy.

Paulus, M. P., \& Stein, M. B. (2010). Interoception in anxiety and depression. Brain Structure and Function, 214(5-6), 451-463. https://doi.org/10.1007/s00429-010-0258-9

Peirce, J., Gray, J. R., Simpson, S., MacAskill, M., Höchenberger, R., Sogo, H., Kastman, E., \& Lindeløv, J. K. (2019). PsychoPy2: Experiments in behavior made easy. Behavior Research Methods, 51(1), 195-203. https://doi.org/10.3758/s13428-018-01193-y

Pennebaker, J. W., \& Hoover, C. W. (1984). Visceral perception versus visceral detection: Disentangling methods and assumptions. Biofeedback and Self-Regulation, 9(3), 339-352. https://doi.org/10.1007/BF00998977

Pernet, C. R., Wilcox, R., \& Rousselet, G. A. (2013). Robust Correlation Analyses: False Positive and Power Validation Using a New Open Source Matlab Toolbox. Frontiers in Psychology, 3. 
https://doi.org/10.3389/fpsyg.2012.00606

Petzschner, F. H., Garfinkel, S. N., Paulus, M. P., Koch, C., \& Khalsa, S. S. (2021). Computational Models of Interoception and Body Regulation. Trends in Neurosciences, 44(1), 63-76. https://doi.org/10.1016/j.tins.2020.09.012

Prins, N. (2012). The psychometric function: The lapse rate revisited. Journal of Vision, 12(6), 25-25. https://doi.org/10.1167/12.6.25

Prins, N., \& Kingdom, F. A. A. (2018). Applying the Model-Comparison Approach to Test Specific Research Hypotheses in Psychophysical Research Using the Palamedes Toolbox. Frontiers in Psychology, 9, 1250. https://doi.org/10.3389/fpsyg.2018.01250

Quintana, D. S., Alvares, G. A., \& Heathers, J. A. J. (2016). Guidelines for Reporting Articles on Psychiatry and Heart rate variability (GRAPH): Recommendations to advance research communication. Translational Psychiatry, 6(5), e803-e803. https://doi.org/10.1038/tp.2016.73

Ring, C., \& Brener, J. (1996). Influence of beliefs about heart rate and actual heart rate on heartbeat counting. Psychophysiology, 33(5), 541-546. https://doi.org/10.1111/j.1469-8986.1996.tb02430.x

Ring, C., \& Brener, J. (2018). Heartbeat counting is unrelated to heartbeat detection: A comparison of methods to quantify interoception. Psychophysiology, 55(9), e13084. https://doi.org/10.1111/psyp.13084

Ring, C., Brener, J., Knapp, K., \& Mailloux, J. (2015). Effects of heartbeat feedback on beliefs about heart rate and heartbeat counting: A cautionary tale about interoceptive awareness. Biological Psychology, 104, 193-198. https://doi.org/10.1016/j.biopsycho.2014.12.010

Rouault, M., McWilliams, A., Allen, M. G., \& Fleming, S. M. (2018). Human Metacognition Across Domains: Insights from Individual Differences and Neuroimaging. Personality Neuroscience, 1, e17. https://doi.org/10.1017/pen.2018.16

Salvatier, J., Wiecki, T. V., \& Fonnesbeck, C. (2016). Probabilistic programming in Python using PyMC3. PeerJ Computer Science, 2, e55. https://doi.org/10.7717/peerj-cs.55

Schandry, R. (1981). Heart Beat Perception and Emotional Experience. Psychophysiology, 18(4), 483-488. https://doi.org/10.1111/j.1469-8986.1981.tb02486.x

Seth, A. K. (2013). Interoceptive inference, emotion, and the embodied self. Trends in Cognitive Sciences, 17(11), 565-573. https://doi.org/10.1016/j.tics.2013.09.007

Seth, A. K., \& Tsakiris, M. (2018). Being a Beast Machine: The Somatic Basis of Selfhood. Trends in Cognitive Sciences, 22(11), 969-981. https://doi.org/10.1016/j.tics.2018.08.008

Sherrington, C. (1952). The integrative action of the nervous system. CUP Archive.

Strigo, A., \& Craig, A. D. (2016). Interoception, homeostatic emotions and sympathovagal balance. Philosophical Transactions of the Royal Society B: Biological Sciences, 371(1708), 20160010. https://doi.org/10.1098/rstb.2016.0010

Vallat, R. (2018). Pingouin: Statistics in Python. Journal of Open Source Software, 3(31), 1026. https://doi.org/10.21105/joss.01026

Valton, V., Wise, T., \& Robinson, O. J. (2020). Recommendations for Bayesian hierarchical model specifications for case-control studies in mental health. ArXiv:2011.01725 [Cs, Stat]. http://arxiv.org/abs/2011.01725

van Bergen, R. S., Ji Ma, W., Pratte, M. S., \& Jehee, J. F. M. (2015). Sensory uncertainty decoded from visual cortex predicts behavior. Nature Neuroscience, 18(12), 1728-1730. https://doi.org/10.1038/nn.4150

van Gent, P., Farah, H., van Nes, N., \& van Arem, B. (2019). HeartPy: A novel heart rate algorithm for the analysis of noisy signals. Transportation Research Part F: Traffic Psychology and Behaviour, 66, 368-378. https://doi.org/10.1016/j.trf.2019.09.015

Warren, C. M., Eldar, E., van den Brink, R. L., Tona, K.-D., van der Wee, N. J., Giltay, E. J., van Noorden, M. S., Bosch, J. A., Wilson, R. C., Cohen, J. D., \& Nieuwenhuis, S. (2016). Catecholamine-Mediated Increases in Gain Enhance the Precision of Cortical Representations. Journal of Neuroscience, 36(21), 5699-5708. https://doi.org/10.1523/JNEUROSCI.3475-15.2016

Waskom, M., Botvinnik, O., Gelbart, M., Ostblom, J., Hobson, P., Lukauskas, S., Gemperline, D. C., Augspurger, T., Halchenko, Y., Warmenhoven, J., Cole, J. B., Ruiter, J. D., Vanderplas, J., Hoyer, S., Pye, C., Miles, A., Corban Swain, Meyer, K., Martin, M., ... Brunner, T. (2020). mwaskom/seaborn: V0.11.0 (Sepetmber 2020) (v0.11.0) [Computer software]. Zenodo. https://doi.org/10.5281/ZENODO.592845

Whitehead, W. E., Drescher, V. M., Heiman, P., \& Blackwell, B. (1977). Relation of heart rate control to heartbeat perception. Biofeedback and Self-Regulation, 2(4), 371-392. https://doi.org/10.1007/BF00998623

Wiecki, T. V., Sofer, I., \& Frank, M. J. (2013). HDDM: Hierarchical Bayesian estimation of the Drift-Diffusion Model in Python. Frontiers in Neuroinformatics, 7. https://doi.org/10.3389/fninf.2013.00014 
bioRxiv preprint doi: https://doi.org/10.1101/2021.02.18.431871; this version posted February 18,2021 . The copyright holder for this

preprint (which was not certified by peer review) is the author/funder, who has granted bioRxiv a license to display the preprint in perpetuity. It is made available under aCC-BY-NC-ND 4.0 International license.

Windmann, S., Schonecke, O. W., Fröhlig, G., \& Maldener, G. (1999). Dissociating beliefs about heart rates and actual heart rates in patients with cardiac pacemakers. Psychophysiology, 36(3), 339-342. https://doi.org/10.1017/S0048577299980381

Zamariola, G., Maurage, P., Luminet, O., \& Corneille, O. (2018). Interoceptive accuracy scores from the heartbeat counting task are problematic: Evidence from simple bivariate correlations. Biological Psychology, 137, 12-17. https://doi.org/10.1016/j.biopsycho.2018.06.006

Zimprich, D., Nusser, L., \& Pollatos, O. (2020). Are interoceptive accuracy scores from the heartbeat counting task problematic? A comment on Zamariola et al. (2018). Biological Psychology, 152, 107868. https://doi.org/10.1016/j.biopsycho.2020.107868 